\title{
多组分反应在合成多取代咪唑类化合物中的应用
}

\author{
肖立伟* 彭晓霞周秋香寇伟时亚茹 \\ (廊坊师范学院 化学与材料科学学院 廊坊 065000)
}

\begin{abstract}
摘要 多取代咪唑类化合物包括三取代咪唑和四取代咪唑, 在医药、化学传感及催化等领域有重要应用. 多组分反应 作为原子经济性和反应选择性很高的一类反应，广泛应用于多取代咪唑的合成中. 基于反应原料及方法进行分类，综 述了近年来利用多组分反应合成多取代咪唑类化合物的研究进展情况.
\end{abstract}

关键词 三取代咪唑; 四取代咪唑; 多组分反应; 异相催化

\section{Application of Multi-component Reaction in Synthesis of Multi- substituted Imidazoles}

\author{
Xiao, Liwei* Peng, Xiaoxia Zhou, Qiuxiang Kou, Wei Shi, Yaru \\ (Faculty of Chemistry and Material Science, Langfang Teachers University, Langfang 065000)
}

\begin{abstract}
Multi substituted imidazoles including tri- and tetra-substituted imidazoles, play important roles in the field of pharmaceutical, chemical sensing and catalysis etc. Multi-component reaction (MCR), a protocol with high atom economic and reaction selectivity, has been applied widly on the synthesis of multi-substituted imidazoles. Based on different starting materials and different methods, the recent advances in the synthesis of multi-substituted imidazols via multi-component reaction are reviewed.
\end{abstract}

Keywords tri-substituted imidazoles; tetra-substituted imidazoles; multi-component reaction; heterogeneous catalysis

咪唑是一类重要的含氮五元杂环化合物，其重要性 不在于其单体，而是其含多种取代基的衍生物. 多取代 咪唑类化合物主要包括三取代咪唑和四取代咪唑，大多 具有强烈的生理性能，如抗真菌、抗调亡、抗病毒、消 炎、抗肿瘤等活性，在医疗及农业领域有广泛的应用 ${ }^{[1,2]}$; 多取代咪唑类化合物还具有优良的光学性能, 应用于苂 光、图像显示、光信息存储、化学传感器及光控开关等 领域 ${ }^{[3,4]}$; 以咪唑盐为主体的离子液体, 具有优良的理化 性能和绿色环保的特性, 在催化、萃取、采矿等领域显 示出良好的应用前景 ${ }^{[5>3}$; 此外, 以咪唑环 (imidazolyliene)为骨架的卡宾 (carbenes), 能够控制和调节与之键 连的金属的配位能力, 成为有机金属催化剂中的一类重 要的配体 ${ }^{[9]}$. 基于多取代咪唑类化合物在多个领域的重 要用途, 其合成方法备受关注.

多组分合成法是合成多取代咪唑类化合物最重要 的方法. 多组分反应(MCR)是将三种或三种以上的原料
“一锅法” 加入反应中, 不经过中间体的分离, 直接获得 结构中含有原料片断的终产物的合成方法. MCR一步即 可构筑起基本的分子骨架或关键的中间体，较传统的分 步合成方法，显示出更高的原子经济性和选择性，因而 在有机合成中应用越来越广泛 ${ }^{[10 ~ 13]}$. 虽然早在 1882 年, Radziszewski ${ }^{[14]}$ 和 Japp 等 ${ }^{[15]}$ 首次利用 1,2 -二酮、醛和 胺一锅法合成了多取代咪唑，但是并没引起人们的关 注. 直到上世纪末，有关多组分反应合成多取代咪唑的 报道才逐渐呈现出快速增长的趋势. 本文以不同的反应 底物及催化剂的类型进行分类，综述了近十多年来多组 分反应在合成多取代咪唑中的应用研究进展情况.

\section{1 以邻二酮或邻羟基酮为底物合成多取代咪唑}

\section{1 以质子酸和 Lewis 酸为催化剂合成多取代咪唑} 质子酸和以金属化合物为代表的 Lewis 酸是有机合

\footnotetext{
* E-mail: xiaoliwei2000@sina.com
}

Received December 5, 2014; revised February 9, 2015; published online February 11, 2015

Project supported by the Natural Science Foundation of Hebei Province (No. B2013408014) and the Key Foundation of Langfang Teachers University (No. LSLZ201402)

河北省自然科学基金(No. B2013408014)和廊坊师范学院重点(No. LSLZ201402)资助项目. 
成中常见的传统催化剂. 在以二酮、醛和乙酸铵(胺)为 原料合成三取代咪唑和四取代咪唑的反应中, 质子酸和 Lewis 酸也是报道最多的一类催化剂. 例如 $\mathrm{NaH}_{2} \mathrm{PO}_{4}{ }^{[16]}$ 、

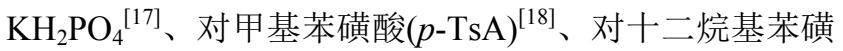
酸(DBSA $)^{[19]}$ 等质子酸和三氯化铟 $\left(\mathrm{InCl}_{3} \cdot 3 \mathrm{H}_{2} \mathrm{O}\right)^{[20] \text { 、三氟 }}$

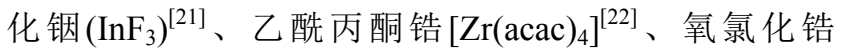
$\left(\mathrm{ZrOCl}_{2} \bullet 8 \mathrm{H}_{2} \mathrm{O}\right)^{[23]}$ 、稀土化合物 $\left[\mathrm{Yb}(\mathrm{OTf})_{3}\right]^{24]}$ 、三价稀土 $\left(\mathrm{Sc} 、 \mathrm{Y} 、 \mathrm{La}-\mathrm{Lu}\right.$ 等)的全氟辛基磺酸盐 $\left[\mathrm{RE}(\mathrm{OPf})_{3}\right]^{[25]}$ 、硝 酸钎 $\left[\mathrm{Y}\left(\mathrm{NO}_{3}\right)_{3} \cdot 6 \mathrm{H}_{2} \mathrm{O}\right]^{[26]}$ 和硝酸铈铵 $\left[\left(\mathrm{NH}_{4}\right)_{2} \mathrm{Ce}\left(\mathrm{NO}_{3}\right)_{6}\right.$, $\mathrm{CAN}]^{[27]}$ 等 Lewis 酸, 这些催化剂在催化合成多取代咪 唑反应中的应用情况见表 1 .

表 1 质子酸和 Lewis 酸催化合成多取代咪唑

Table 1 Synthesis of multi-substituted imidazoles catalyzed by proton acid and Lewis acid
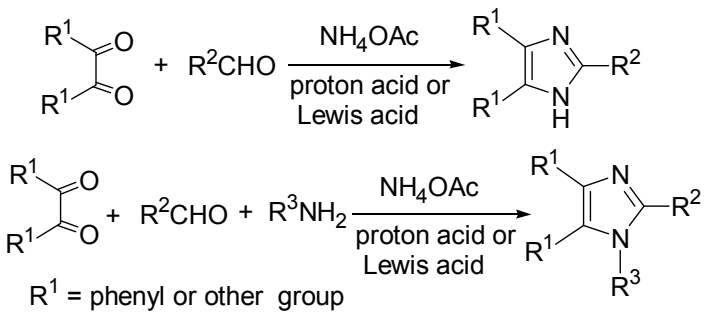

$\mathrm{R}^{2}=$ phenyl or other aryl group; $\mathrm{R}^{3}=$ aryl or alkyl

\begin{tabular}{|c|c|c|c|c|}
\hline Entry & Catalyst & Yield $^{a} / \%$ & Yield $^{b} / \%$ & Ref. \\
\hline 1 & $\mathrm{NaH}_{2} \mathrm{PO}_{4}$ & $98 \sim 99$ & $80 \sim 92$ & [16] \\
\hline 2 & $\mathrm{KH}_{2} \mathrm{PO}_{4}$ & $88 \sim 93$ & - & [17] \\
\hline 3 & $p$-TsA & - & $80 \sim 92$ & [18] \\
\hline 4 & DBSA & $71 \sim 89$ & $73 \sim 86$ & [19] \\
\hline $5^{c}$ & $\mathrm{InCl}_{3} \cdot 3 \mathrm{H}_{2} \mathrm{O}$ & $54 \sim 82$ & $47 \sim 84$ & [20] \\
\hline $6^{d}$ & $\mathrm{InF}_{3}$ & $80 \sim 92$ & - & [21] \\
\hline $7^{e}$ & $\mathrm{Zr}(\mathrm{acac})_{4}$ & 82 & - & [22] \\
\hline $8^{f}$ & $\mathrm{ZrOCl}_{2} \bullet 8 \mathrm{H}_{2} \mathrm{O}$ & $92 \sim 97$ & - & [23] \\
\hline 9 & $\mathrm{Yb}(\mathrm{OTf})_{3}$ & $\begin{array}{l}\mathrm{R}^{2}=\mathrm{Ar} \\
73 \sim 97 \\
\mathrm{R}^{2}=\text { alky } \\
17 \sim 24\end{array}$ & - & [24] \\
\hline 10 & $\mathrm{Yb}(\mathrm{OPf})_{3} \cdot 6 \mathrm{H}_{2} \mathrm{O}$ & $\begin{array}{l}\mathrm{R}^{2}=\mathrm{Ar}, \\
7 \sim 97 ; \\
\mathrm{R}^{2}=\text { alky, } \\
10 \sim 31\end{array}$ & - & [25] \\
\hline 11 & $\mathrm{Y}\left(\mathrm{NO}_{3}\right)_{3}$ & $70 \sim 98$ & - & [26] \\
\hline 12 & CAN & $85 \sim 96$ & - & [27] \\
\hline
\end{tabular}

${ }^{a}$ Yield for tri-substituted products; ${ }^{b}$ Yield for tetra-substituted products; ${ }^{c} \mathrm{R}^{1}=$ $\mathrm{Ph}, 4-\mathrm{CH}_{3} \mathrm{C}_{6} \mathrm{H}_{4} ;{ }^{d} \mathrm{R}^{1}=4-\mathrm{FC}_{6} \mathrm{H}_{4}, \mathrm{C}_{6} \mathrm{H}_{5} ;{ }^{e} \mathrm{R}^{1}=\mathrm{Ph}, 4-\mathrm{NO}_{2} \mathrm{C}_{6} \mathrm{H}_{4}, 4-\mathrm{FC}_{6} \mathrm{H}_{4} ;{ }^{f} \mathrm{R}^{1}=$ $\mathrm{Ph}, 4-\mathrm{ClC}_{6} \mathrm{H}_{4}$.

苯偶酰是合成多取代咪唑中使用最多的邻二酮, 合 成四取代咪唑与合成三取代咪唑相比, 除了原料中增加 伯胺外, 其他操作相似. 由表 1 可知, 质子酸和 Lewis 酸催化合成多取代咪唑具有操作简单、效果好, 适用范 围宽，大多数含不同取代基的反应底物均可以获得良好
的收率. 反应中的所用的醛主要是芳醛，选用脂肪醛往 往收率较低(Table 1, Entry 9) ${ }^{[24,25]}$, 在合成多取代咪唑的 原料中，1,2-二酮(如苯偶酰)还可以用邻羟基酮(如苯偶 姻)替代, 多数情况下, 收率会略有下降 ${ }^{[23]}$. 合成多取代 咪唑时, 醛或胺可以选择杂环醛, 如呋喃醛(Table 1, Entry 8$)^{[23]}$ 、吲哚醛(Table 1, Entry 8) ${ }^{[26]}$ 等, 以及杂环胺, 如异噁唑胺(Table 1, Entry 12) ${ }^{[27]}$ 等(Scheme 1), 这些物 质大多具有特定的生物活性.
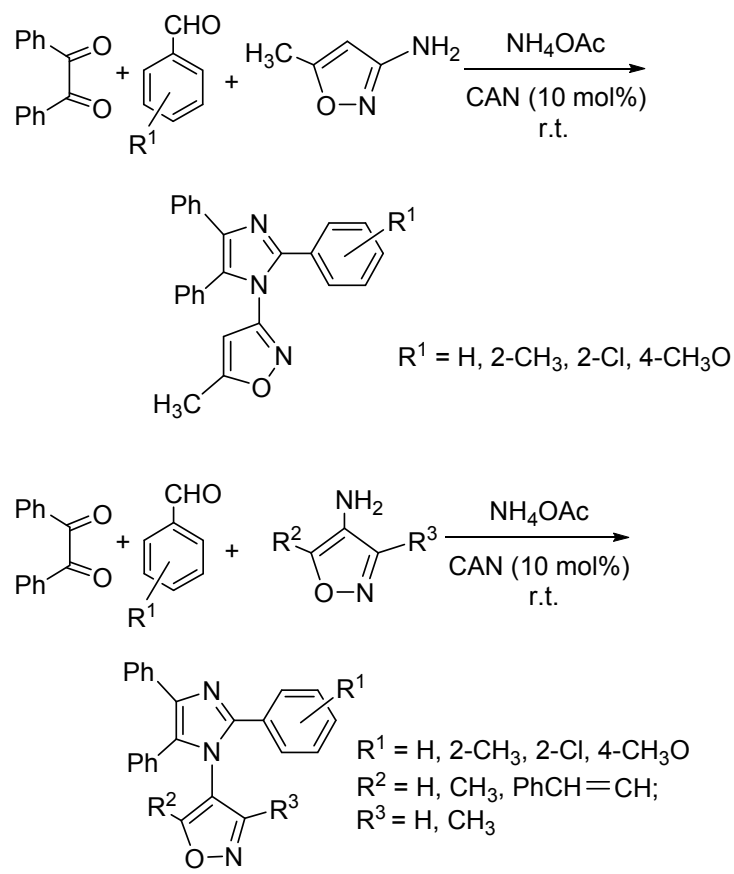

图式 1 杂环胺用于合成四取代咪唑

Scheme 1 Synthesis of tetra-substituted imidazoles from heterocyclic amines

质子酸和 Lewis 酸催化 1,2-二酮与醋酸铵及醛、胺 通过多组分反应生成三取代及四取代咪唑，具有类似地 反应机理：质子酸和 Lewis 酸提供空轨道，反应底物官 能团中的孤对电子填充到空轨道中, 使反应底物活化. 以 $\mathrm{InCl}_{3} \cdot 3 \mathrm{H}_{2} \mathrm{O}$ 催化苯偶酰、胺、醛和乙酸铵合成三取代 咪唑为例说明。醋酸铵分解生成氨和醋酸; 在 $\mathrm{InCl}_{3} \cdot 3 \mathrm{H}_{2} \mathrm{O}$ 活化下, 芳醛与 2 倍物质的量的氨作用形成 偕二胺中间体 $\mathbf{a}$; 被 $\mathrm{InCl}_{3} \cdot 3 \mathrm{H}_{2} \mathrm{O}$ 活化的苯基乙二酮与 $\mathbf{a}$ 作用脱去 2 分子的水形成双亚胺五元环 $\mathbf{b} ; \mathbf{b}$ 经氢原子 $[1,5]$ 迁移重排得产物三取代咪唑 c (Scheme 2). 四取代 咪唑的形成机理与三取代咪唑类似: 在 $\mathrm{InCl}_{3} \cdot 3 \mathrm{H}_{2} \mathrm{O}$ 活化 下，醛与等物质的量的伯胺和氨作用形成中间体 $\mathbf{a} ; \mathbf{a}$ 与 活化的二酮作用脱去 1 分子水形成双亚胺五元环 $\mathbf{b} ; \mathbf{b}$ 重 排脱去一分子水得四取代咪唑 $\mathbf{c}$ (Scheme 3).

\section{2 以不含金属的催化剂催化合成多取代咪唑}

化学反应中使用的催化剂，多数含有金属元素，近 


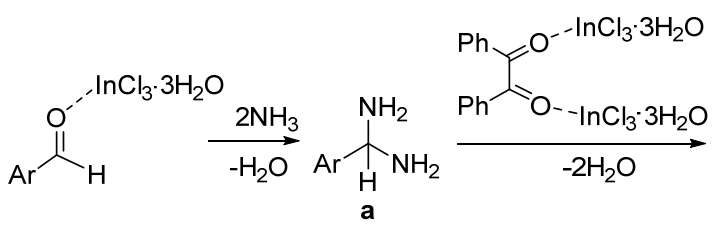

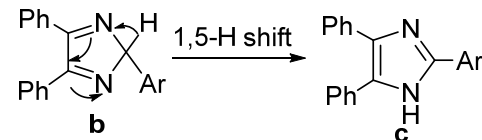

图式 2 氯化铟催化合成三取代咪唑的反应机理

Scheme 2 The mechanism in formation tri-substituted imidazoles catalyzed by $\mathrm{InCl}_{3} \cdot 3 \mathrm{H}_{2} \mathrm{O}$

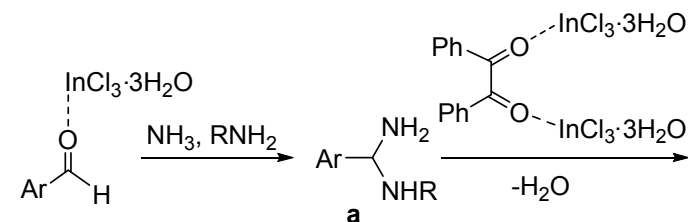<smiles>[R]n1c([Al])nc(-c2ccccc2)c1-c1ccccc1</smiles>

图式 3 氯化铟催化合成四取代咪唑的反应机理

Scheme 3 The mechanism in formation tetra-substituted imidazoles catalyzed by $\mathrm{InCl}_{3} \cdot 3 \mathrm{H}_{2} \mathrm{O}$

年来不含金属的催化剂如非金属、有机催化剂 (Organocatalysts)等已经引起人们的关注. 非金属或有机 催化剂在催化合成三取代咪唑和四取代咪唑的反应中 应用也越来越普遍, 如分子碘 ${ }^{[28]}$ 、聚乙二醇 400 (PEG-

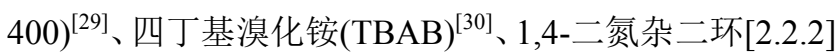

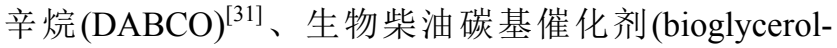
based carbon catalyst $)^{[32]} 、 L$-脯氨酸 $(L \text {-Proline })^{[33]}$, 溴代磷 酸二乙酯(DEP, diethyl bromophosphate $)^{[34]}$ 和醋酸 ${ }^{[35]}$, 这 些催化剂具有催化效果好, 适用范围宽, 对环境友好等 优点, 具体情况如表 2 所示.

不含金属的催化剂催化苯偶酰、胺、醛和乙酸铵(胺) 合成多取代咪唑的机理与质子酸和 Lewis 酸的催化机 理类似. 以分子碘催化三取代取代咪唑的合成为例. 醋 酸铵分解生成氨和醋酸; 在分子碘活化下, 芳醛与 2 倍 物质的量的氨作用形成偕二胺中间体 $\mathbf{a}$; 被分子碘活化 的苯基乙二酮与 $\mathbf{a}$ 作用形成中间体 $\mathbf{b}$, 进而脱去 2 分子 水形成双亚胺五元环(异咪唑) $\mathbf{c}$; $\mathbf{c}$ 经氢原子 [1,5]迁移重 排得产物三取代咪坐 d (Scheme 4).

反应底物除常见的苯偶酰或苯偶姻之外, 还有其他 邻二酮类化合物. 如含有咪唑杂环取代基的双酮与醛、 醋酸铵，在醋酸作用下反应，生成一系列不同取代的 4,4-联咪唑类化合物(Eq. 1) ${ }^{[36]}$, 产率为 $71 \% \sim 96 \%$. 合成
表 2 不含金属的催化剂催化合成多取代咪唑

Table 2 Synthesis of multi-substituted imidazoles catalyzed by metal-free catalyst
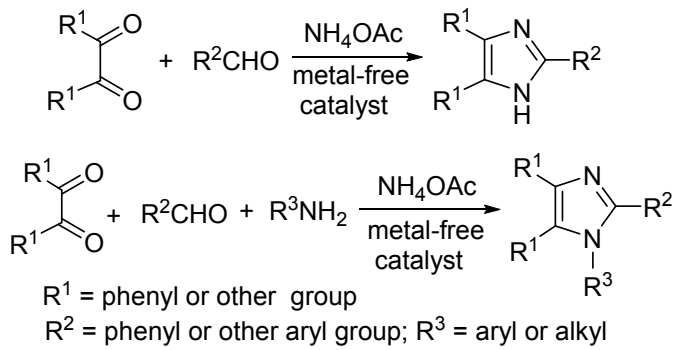

\begin{tabular}{|c|c|c|c|c|}
\hline Entry & Catalyst & Yield $^{a} / \%$ & Yield $^{b} / \%$ & Ref. \\
\hline 1 & $\mathrm{I}_{2}$ & $97 \sim 99$ & $97 \sim 99$ & [28] \\
\hline 2 & PEG-400 & $82 \sim 93$ & $78 \sim 90$ & [29] \\
\hline 3 & TBAB & $72 \sim 92$ & - & [30] \\
\hline $4^{c}$ & DABCO & $72 \sim 92$ & $70 \sim 82$ & [31] \\
\hline $5^{d}$ & $\begin{array}{l}\text { Bioglycerol } \\
\text { based carbon } \\
\text { catalyst }\end{array}$ & $80 \sim 85$ & $70 \sim 80$ & [32] \\
\hline $6^{e}$ & $L$-Proline & $\begin{array}{l}\mathrm{R}^{2}=\text { alkyl, } \\
40 \sim 56 \\
\mathrm{R}^{2}=\mathrm{Ar} \\
75 \sim 90\end{array}$ & $78 \sim 86$ & [33] \\
\hline 7 & DEP & $90 \sim 97$ & - & [34] \\
\hline 8 & $\mathrm{AcOH}$ & - & 62,72 & [35] \\
\hline
\end{tabular}

${ }^{a}$ Yield for tri-substituted products; ${ }^{b}$ Yield for tetra-substituted products; ${ }^{c} \mathrm{R}^{1}=$ $\mathrm{Ph}, 4-\mathrm{CH}_{3} \mathrm{C}_{6} \mathrm{H}_{4}, 3$-furyl; ${ }^{d} \mathrm{R}^{1}=\mathrm{Ph}, 4-\mathrm{MeC}_{6} \mathrm{H}_{4} ;{ }^{e} \mathrm{R}^{1}=\mathrm{Ph}, 4-\mathrm{ClC}_{6} \mathrm{H}_{4}, 4-\mathrm{MeO}-$ $\mathrm{C}_{6} \mathrm{H}_{4}$.

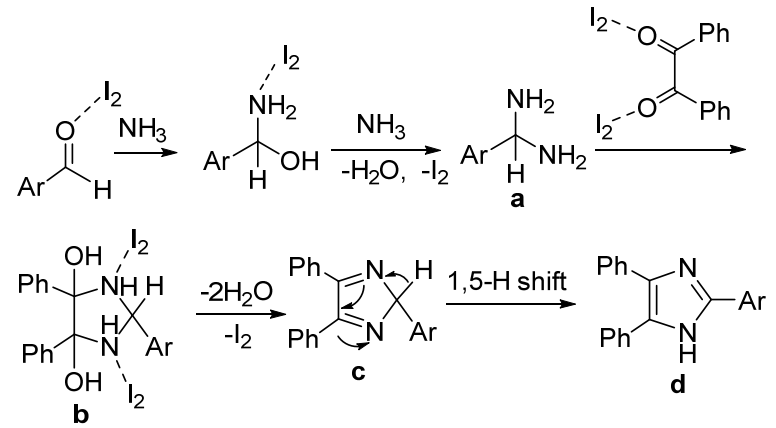

图式 4 单质碘催化合成三取代咪唑的反应机理

Scheme 4 The mechanism in formation of tri-substituted imidazoles catalyzed by iodine

的化合物作为菼光及发色传感器可用于检测重金属离 子，其中， 2 和 $2^{\prime}$ 位的取代基可以不同，为合成非对称结 构联杂环类功能材料提供了一种新颖的方法.

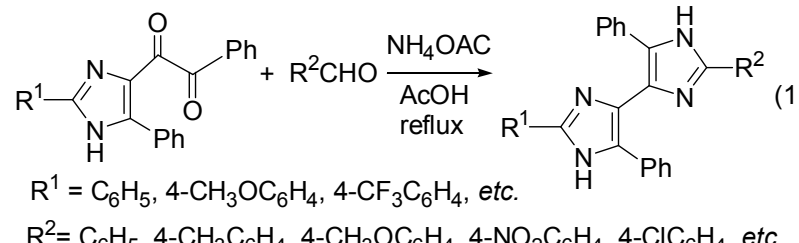


在氮源的存在下, 醛与邻二酮作用, 生成多取代咪 唑，酮也会发生上述反应，但是反应的机理却有所相同. 如 Xue 等 ${ }^{[37]}$ 以芳香酮、苯偶姻、醋酸铵为原料, 在碘碳酸铯体系催化下, 合成了含有羰基的三取代咪唑(Eq. 2). 虽然整个反应过程经过了若干步骤, 但是反应中投 料采用一锅法进行, 因而, 该方法发展了一种新颖的合 成含羰基的多取代咪唑类化合物的方法.

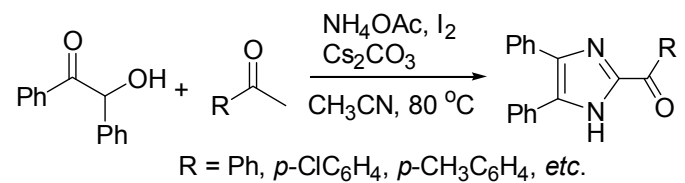

该反应可能的机理如下(Scheme 5): 在强碱存在下, 苯乙酮与碘作用形成 $\alpha$-碘代苯乙酮 $\mathbf{a}$; 苯偶姻被碘氧化 成为苯偶酰, 苯偶酰与醋酸铵分解放出的氨作用生成双 亚胺化合物 $\mathbf{b}$; 双亚胺化合物与 $\alpha$-碘代苯乙酮作用, 经 过互变异构, 发生分子内亲核反应形成五元环二氢咪唑 类化合物 $\mathbf{c}$; 进一步在碘氧化下发生脱氢反应得产物多 取代咪唑 d.<smiles>CC(=O)c1ccccc1</smiles>

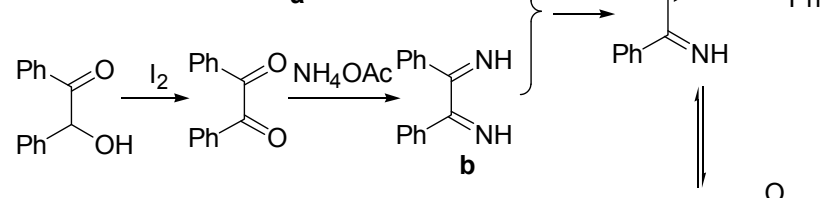<smiles>CC(C)(C)c1ccccc1-c1ccccc1</smiles>

图式 5 以苯偶姻及苯乙酮为原料合成三取代咪唑的反应机 理

Scheme 5 The mechanism in formation of tri-substituted imidazoles from benzoin and acetophenone

\section{3 以离子液体为催化剂合成多取代咪唑}

离子液体是指在室温或接近室温下呈现液态、由阴 阳离子组成的盐, 也称为低温熔融盐. 离子液体具有蒸 汽压低、热稳定性高的特点, 对很多有机及无机化合物 具有较好的溶解性, 是一种优良的反应介质和催化剂. 近年来, 在多取代咪唑类化合物的合成中, 采用离子液 体作催化剂越来越普遍. 已报道的离子液体有 1-乙基-3-

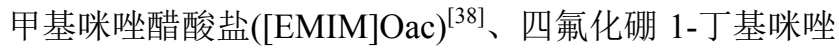
盐 $\left([\mathrm{Hbim}] \mathrm{BF}_{4}\right)^{[39]}$ 、四氟化嗍 1 -甲基-3-庚基咪唑盐 $\left([\mathrm{HeMIM}] \mathrm{BF}_{4}\right)^{[40]} 、 N$-甲基-2-吡咯烷酮硫酸氢盐([NMP]$\left.\mathrm{HSO}_{4}\right)^{[41]} 、 1,3$-吡啶二磺酸硫酸氢盐 $\left([\mathrm{Dsim}] \mathrm{HSO}_{4}\right)^{[42]} 、 1$ 甲基咪唑三氟乙酸盐([Hmim $] \mathrm{TFA})^{[43]}$, 以及负载型离子
液体 SILLP ${ }^{[44]}$ 等. 部分离子液体的结构如图 1 所示. 离 子液体用于催化合成多取代咪唑的详细情况见表 3 .

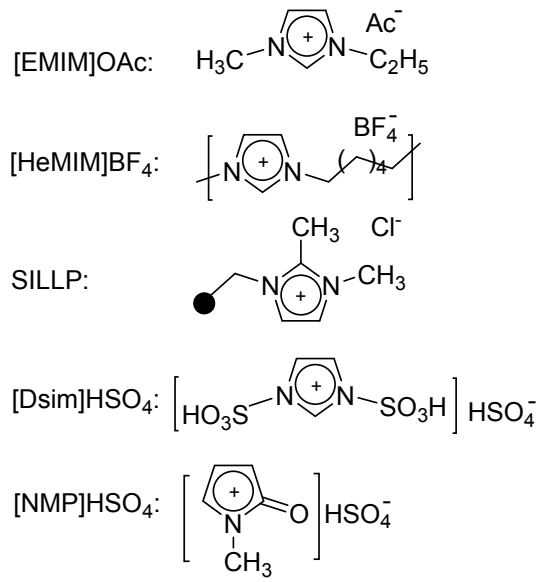

图 1 部分离子液体的结构

Figure 1 The structure of some ionic liquids

表 3 离子液体催化剂催化合成多取代咪唑

Table 3 Synthesis of multi-substituted imidazoles catalyzed by ionic liquids
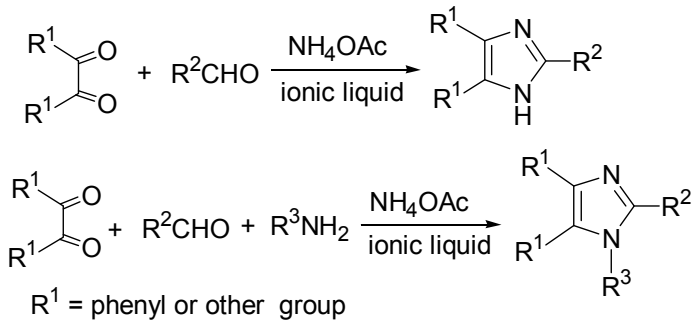

$\mathrm{R}^{2}=$ phenyl or other aryl group; $\mathrm{R}^{3}=$ aryl or alkyl

\begin{tabular}{clccc}
\hline Entry & \multicolumn{1}{c}{ Catalyst } & Yield $^{a} \%$ & Yield $^{b} \%$ & Ref. \\
\hline 1 & {$[$ EMIM]OAc } & $71 \sim 96$ & - & {$[38]$} \\
$2^{c}$ & {$\left[\mathrm{Hbim}_{\mathrm{B}} \mathrm{BF}_{4}\right.$} & $85 \sim 98$ & - & {$[39]$} \\
3 & {$[\mathrm{HeMIM}] \mathrm{BF}_{4}$} & $74 \sim 83$ & - & {$[40]$} \\
4 & {$[\mathrm{NMP}] \mathrm{HSO}_{4}$} & $80 \sim 98$ & $80 \sim 98$ & {$[41]$} \\
5 & {$[\mathrm{Dsim}] \mathrm{HSO}_{4}$} & - & $75 \sim 100$ & {$[42]$} \\
6 & {$[\mathrm{Hmim}] \mathrm{TFA}$} & $90 \sim 95$ & - & {$[43]$} \\
7 & SILLP $^{4}$ & - & $85 \sim 90$ & {$[44]$} \\
\hline
\end{tabular}

${ }^{a}$ Yield for tri-substituted products; ${ }^{b}$ Yield for tetra-substituted products; ${ }^{c} \mathrm{R}^{1}=$ $\mathrm{Ph}, o$-furyl, $p$-tolyl.

离子液体作为一种高效、绿色的反应介质，具有操 作过程简单，催化效率高等优点，反应结束后，催化剂 很容易从反应体系中分离出来, 重新使用. 在离子液体

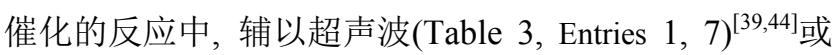
微波辐射(Table 3, Entry 3) ${ }^{[40]}$, 会大幅度缩短反应时间, 如以二醛为原料，在微波辐射 $2 \sim 6 \mathrm{~min}$, 在中性离子液 体 $[\mathrm{HeMIM}] \mathrm{BF}_{4}$ 中合成双三取代咪唑衍生物 ${ }^{[40]}($ Scheme 6). 该方法综合了微波辐照和离子液体二者的优点, 使 反应污染低、速度快、后处理简单.

合成多取代咪唑时, 反应底物除苯偶酰或苯偶姻之 外，还有其他邻二酮类化合物。如 9,10-邻菲罗啉二 
<smiles>CC(=O)ONC(=O)c1ccc(C=O)cc1</smiles><smiles></smiles><smiles>C=CC(=O)Oc1ccc(C=O)cc1</smiles>

图式 6 微波促进离子液体催化合成多取代咪唑类化合物 Scheme 6 Synthesis of multi-substituted imidazoles catalyzed by ionic liguid under microwave irradiation

酮 ${ }^{[44]}$. 在负载型离子液体 SILLP 催化下, 以苯偶酰或 9, 10 -邻菲罗啉二酮为二酮反应底物, 在超声波辐射条件 下高产率(89\% 94\%)地合成了三取代咪唑类化合物 (Eq. 3).

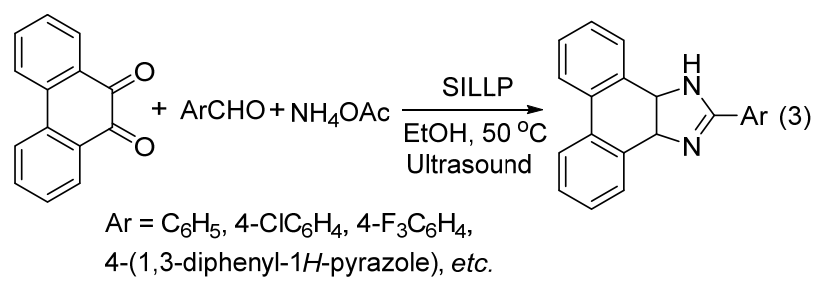

\section{5 以杂多酸为催化剂合成多取代咪唑}

杂多酸(HPA)作为催化剂, 具有催化效率高, 环境 友好等优点, 其中, Keggin 型杂多酸是最常见的一类杂 多酸. 例如, 杂多酸 $\mathrm{H}_{4}\left[\mathrm{PMo}_{11} \mathrm{VO}_{40}\right]^{[45]}$ 对合成四取代咪 唑具有良好的催化作用(Eq. 4), 反应原料在乙醇中回流 5 33 min, 产率达 68\% 90\%. 该催化剂适用面广, 不 论脂肪胺还是芳香胺均可以顺利得到目标产物, 但是芳 胺比脂肪胺产率明显高.

$$
\mathrm{C}_{\mathrm{O}}^{\mathrm{O}}+\mathrm{R}^{1} \mathrm{CHO}+\mathrm{R}^{2} \mathrm{NH}_{2} \underset{\mathrm{EtOH} / \text { reflux }}{\mathrm{HPA}} \stackrel{\mathrm{NH}_{4} \mathrm{OAc}}{\mathrm{HPA}}
$$

$\mathrm{R}^{1}=\mathrm{Ph}, 4-\mathrm{CH}_{3} \mathrm{C}_{6} \mathrm{H}_{4}, 4-\mathrm{BrC}_{6} \mathrm{H}_{4} ; \mathrm{R}^{2}=\mathrm{CH}_{3}, \mathrm{Ph}, \mathrm{PhCH}_{2}$

类似地, 苯偶酰、醛、胺以及醋酸铵反应在三水合 钴铇酸钾 $\left(\mathrm{PDTC}, \mathrm{K}_{5} \mathrm{CoW}_{12} \mathrm{O}_{40} \cdot 3 \mathrm{H}_{2} \mathrm{O}\right)$ 催化下, 在无溶剂 及微波辐射下反应 $2 \mathrm{~min}$, 合成四取代咪唑化合物, 产 率达 $85 \% \sim 95 \%{ }^{[46]}$. 反应中如果采用苯偶姻为反应底物, 反应仍能顺利进行, 但是反应时间相对较长, 产率略有 降低. 此外, 2010 年, Fantini 等 ${ }^{[47]}$ 通过甲氧基或邻羟基
苯甲醛与 1,2 二酮化合物及乙酸铵作用，微波照射 10 $20 \mathrm{~min}$, 获得含有咪唑环的苯并噁嗪多杂环化合物 (Scheme 7), 产率达 75\% 90\% 。该方法对合成含有咪唑 骨架的多环药物具有重要意义. 上述反应过程在普通加 热条件下进行，产率只有 $50 \% \sim 85 \%$, 反应时间则需要 16 24h，显示了微波化学的优势.

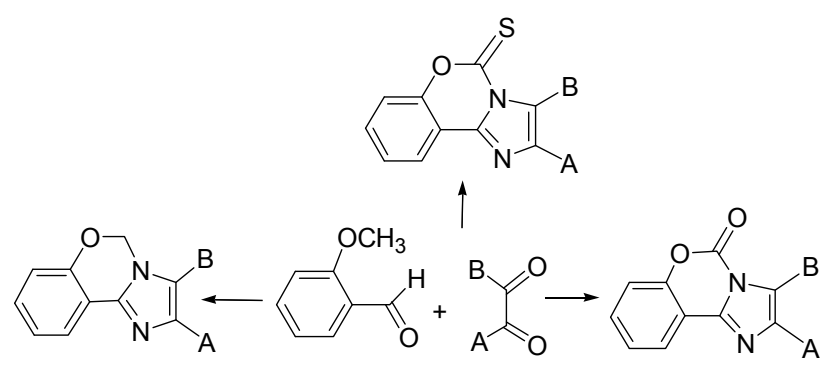

$\mathrm{A}, \mathrm{B}=\mathrm{H}, \mathrm{CH}_{3}, n-\mathrm{CH}_{2} \mathrm{CH}_{2} \mathrm{CH}_{3}, \mathrm{Ph}$

图式 7 含咪唑骨架的稠环化合物的合成

Scheme 7 Synthesis of fused ring compounds containing imidazole frames

\section{6 普通无机及高分子材料负载型催化剂催化合成多 取代咪唑}

异相催化剂一般是把活性成分负载到无机或有机 高分子材料上而形成的负载型催化剂. 异相催化技术的 应用，使反应产物与催化体系容易分离，催化剂容易回 收循环使用，提高了催化剂的利用率。近年来，有关负 载型催化剂用于催化合成三取代咪唑和四取代咪唑的 报道越来越多, 例如, 酸性氧化铝负载氯化镍 $\left(\mathrm{Al}_{2} \mathrm{O}_{3}\right.$ ・

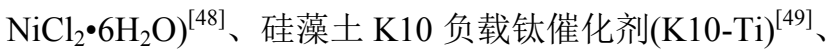
硅胶负载高氯酸 $\left(\mathrm{SiO}_{2}-\mathrm{HClO}_{4}\right)^{[50]}$ 、硅胶负载硫酸氢钠 $\left(\mathrm{SiO}_{2}-\mathrm{NaHSO}_{4}\right)^{[51]} 、 3$-哌嗪- $N$-丙基硅烷(3-PNPS) 负载氯 磺酸 (SBPPSA $)^{[52]}$ 、改性硅胶负载 (偶联) 硫醇 (mercaptopropylsilica, MPS $)^{[53]}$ 、沸石负载硝酸铜 $\left[\text { Zeoliet- } \mathrm{Cu}\left(\mathrm{NO}_{3}\right)_{2}\right]^{[54]}$ 、磺化淀粉或硅胶负载型 $\mathrm{AlCl}_{3}$ $\left[\mathrm{CSC}-\mathrm{star}-\mathrm{SO}_{3}-\mathrm{AlCl}_{2}\right]^{[55]}$ 、含磺酸基的聚合物 poly-

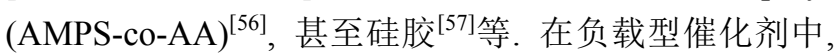
活性成分和载体之间的相互作用既可以是简单的物理 吸附, 还可以通过化学键相连. 部分负载型催化剂的结 构如图 2 所示. 最近, 作者制备了改性活性炭负载氯化

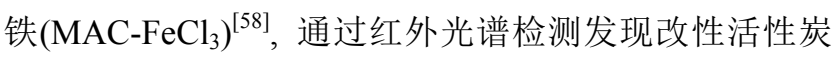
与 $\mathrm{FeCl}_{3}$ 之间存在较强的配位作用, 经测定 $\mathrm{MAC}-\mathrm{FeCl}_{3}$ 中所含含铁量为 $2.1 \mathrm{mmol} / \mathrm{g}$, 催化剂对合成多取代咪唑 具有很好的催化作用，产率达 79\% 91\%. 负载型催化 剂催化合成多取代咪唑的详细情况见表 4 .

从表 4 可以看出, 负载型催化剂对含不同取代基的 醛及胺为原料合成多取代咪唑均具有较好的催化作用. 在合成多取代咪唑的原料中，将苯偶酰换用苯偶姻反 


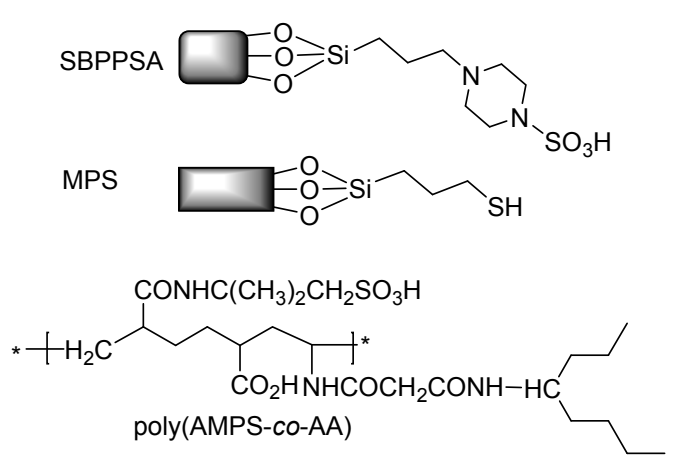

图 2 部分负载催化剂的结构

Figure 2 The structure of some supported catalyst

表 4 负载型催化剂催化合成多取代咪唑

Table 4 Synthesis of multi-substituted imidazoles catalyzed by supported catalyst

$$
\begin{aligned}
& \mathrm{R}^{1}=\text { phenyl or other group } \\
& \mathrm{R}^{2}=\text { phenyl or other aryl group; } \mathrm{R}^{3}=\text { aryl or alkyl }
\end{aligned}
$$

\begin{tabular}{|c|c|c|c|c|}
\hline Entry & Catalyst & Yield $^{a} / \%$ & Yield $^{b} / \%$ & Ref. \\
\hline 2 & $\mathrm{Al}_{2} \mathrm{O}_{3}-\mathrm{NiCl}_{2}$ & $86 \sim 94$ & - & [48] \\
\hline 3 & $\mathrm{~K} 10-\mathrm{Ti}$ & - & $71 \sim 84$ & [49] \\
\hline $4^{c}$ & $\mathrm{SiO}_{2}-\mathrm{HClO}_{4}$ & - & $75 \sim 96$ & [50] \\
\hline 5 & $\mathrm{SiO}_{2}-\mathrm{NaHSO}_{4}$ & - & $\begin{array}{l}88 \sim 89 \text { (Benzi) } \\
20 \sim 30 \text { (Benzoinl) }\end{array}$ & {$[51]$} \\
\hline 6 & SBPPSA & - & $72 \sim 92$ & {$[52]$} \\
\hline $7^{d}$ & MPS & $65 \sim 96$ & $84 \sim 93$ & [53] \\
\hline 8 & Zeolite- $\mathrm{Cu}\left(\mathrm{NO}_{3}\right)_{2}$ & $71 \sim 98$ & $\begin{array}{l}63 \sim 92(\text { Benzil }) \\
43 \sim 84 \text { (Benzoinl) }\end{array}$ & [54] \\
\hline 9 & $\mathrm{CSC}$-star- $\mathrm{SO}_{3}-\mathrm{AlCl}_{2}$ & - & $82 \sim 96$ & {$[55]$} \\
\hline 10 & Poly(AMPS-co-AA) & - & $85 \sim 90$ & {$[56]$} \\
\hline 11 & $\mathrm{SiO}_{2}$ & - & $82 \sim 93$ & [57] \\
\hline 12 & MAC- $-\mathrm{FeCl}_{3}$ & $79 \sim 91$ & $81 \sim 90$ & [58] \\
\hline
\end{tabular}

${ }^{a}$ Yield for tri-substituted products; ${ }^{b}$ Yield for tetra-substituted products; ${ }^{c} \mathrm{R}^{1}=$ $\mathrm{Ph}, \mathrm{CH}_{3} ;{ }^{d} \mathrm{R}^{1}=\mathrm{Ph}, \mathrm{CH}_{3}$, phenanthryl.

应，反应时间需延长，而产率也会有所降低 ${ }^{[51]}$ (Table 4, Entries 5,8). 太阳能取之不竭, 不会产生环境污染, 是 一种理想的清洁能源. 将太阳能引入化学反应中((Table 4 , Entry 11 $)^{[57]}$, 在实验室实现太阳能向化学能的转化, 是很好的一次尝试, 具有潜在的应用价值. 反应的底物 除苯偶酰或苯偶姻之外, 还可以是其他邻二酮类化合 物, 如 9,10-菲二酮 ${ }^{[53]}$, 以改性硅胶负载硫醇 $\mathrm{MPSz}$ 为催 化剂，通过 9,10-菲二酮、芳醛、芳胺及醋酸铵间反应，高 产率地合成了三取代、四取代咪唑类化合物(Eq. 5). 该 方法反应条件温和, 催化剂能多次循环使用, 仍保持较 高的催化活性.

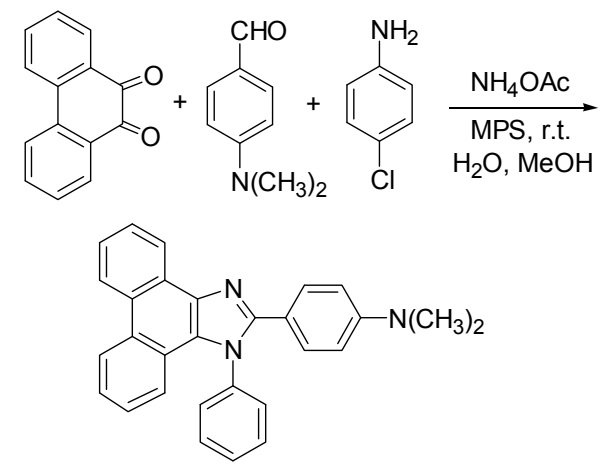

\section{7 纳米催化剂及介孔材料催化剂催化合成多取代咪 唑}

由于纳米材料具有与宏观物质迥异的表面效应和 小尺寸效应等特性, 具有特殊的光、电、磁、催化等性 能. 本文提到的纳米催化剂分为两类, 即纳米材料本身 作催化剂和纳米材料负载活性成分作催化剂. 2013 年, Safari 等合成了一系列纳米材料负载催化剂, 如超顺磁 $\mathrm{Fe}_{3} \mathrm{O}_{4}$ 纳米颗粒负载离子液体 1-甲基-3-(3-甲氧基硅基丙 基)咪唑盐酸盐(IL-MNPs) ${ }^{[59]}$ 、磁性 $\mathrm{Fe}_{3} \mathrm{O}_{4}$ 纳米颗粒负载 氯磺酸(SA-MNPs) ${ }^{[60]}$ 、纳米晶状 $\mathrm{MgAl}_{2} \mathrm{O}_{4}{ }^{[61]}$ 等, 这些纳 米催化剂对合成多取代咪唑类化合物, 具有良好的催化 活性. 此外, 还有纳米晶状硫酸锆 $\left(\mathrm{Zr}\left(\mathrm{SO}_{4}\right)_{2}\right)^{[62]}$ 、多孔硅 基纳米材料负载有机酸(PSNP-CA) ${ }^{[63]}$ 、水合氧化铝(水软 石)纳米颗粒 $\mathrm{AlOOH} \mathrm{NPs}{ }^{[64]}$ 、纳米 $\mathrm{SiO}_{2}$ 负载 $\mathrm{TiCl}_{4}{ }^{[65]}$, 共 融混合物 $\mathrm{Fe}_{3} \mathrm{O}_{4}$ 纳米颗粒 ${ }^{[66]}$. 部分纳米催化剂的结构如 图 3 所示. 除了纳米催化剂外, 具有规则孔洞结构的介 孔材料也用于催化合成多取代咪唑, 如 SBA-15 和 2,2,2三氟乙醇组成的非金属催化体系(SBA-15/TFE) ${ }^{[67]}$.

由表 5 可知, 无论纳米材料本身作催化剂或纳米材 料负载活性成分作催化剂, 均具有较高的催化活性. 在

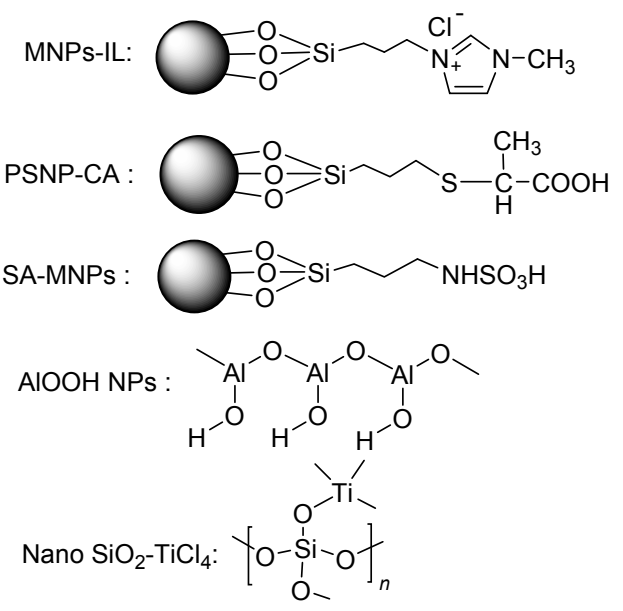

图 3 部分纳米催化剂的结构

Figure 3 The structure of some nano catalyst 
表 5 纳米催化剂催化合成多取代咪唑

Table 5 Synthesis of multi-substituted imidazoles catalyzed by nano-catalyst
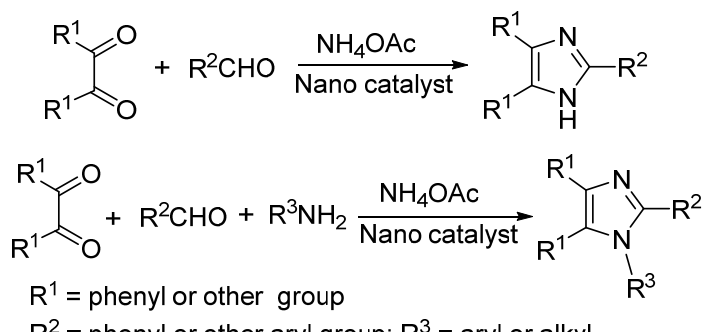

$\mathrm{R}^{2}=$ phenyl or other aryl group; $\mathrm{R}^{3}=$ aryl or alkyl

\begin{tabular}{|c|c|c|c|c|}
\hline Entry & Catalyst & Yield $^{a} / \%$ & Yield $^{b} / \%$ & Ref. \\
\hline 1 & IL-MNPs & - & $80 \sim 98$ & [59] \\
\hline $2^{c}$ & SA-MNPs & $90 \sim 98$ & - & {$[60]$} \\
\hline 3 & $\begin{array}{l}\mathrm{MgAl}_{2} \mathrm{O}_{4} \\
\text { nano-crystaline }\end{array}$ & - & $89 \sim 95$ & {$[61]$} \\
\hline 4 & $\begin{array}{l}\mathrm{Zr}\left(\mathrm{SO}_{4}\right)_{2} \\
\text { nano-crystalin }\end{array}$ & $82 \sim 93$ & $73 \sim 93$ & {$[62]$} \\
\hline $5^{d}$ & PSNP-CA & - & $78 \sim 97$ & {$[63]$} \\
\hline 6 & $\begin{array}{l}\mathrm{AlOOH} \\
\text { NPs nanoparticle }\end{array}$ & $79 \sim 98$ & $78 \sim 90$ & {$[64]$} \\
\hline 7 & Nano $\mathrm{SiO}_{2}-\mathrm{TiCl}_{4}$ & - & $60 \sim 90$ & {$[65]$} \\
\hline $8^{e}$ & $\begin{array}{l}\mathrm{Fe}_{3} \mathrm{O}_{4} \text { eutectic } \\
\text { nano particle }\end{array}$ & $60 \sim 80$ & $68 \sim 90$ & {$[66]$} \\
\hline 9 & SBA-15/TFE & - & $80 \sim 90$ & [67] \\
\hline
\end{tabular}

纳米催化剂促进的反应中, 辅以微波辐射(Table 4, Entry 1) ${ }^{[59]}$ 或用超声波辐射(Table 4, Entries 2, 3) ${ }^{[60,61]}$, 在产率 相当时, 会使反应时间缩短数倍.

\section{2 由二酮的衍生物为底物合成多取代咪唑}

近些年，过渡金属及其化合物引发的交叉偶联反 应, 引起了人们的广泛关注. 最近, Rajaguru等 ${ }^{[68]}$ 在稀土 化合物三氟甲基磺酸铒催化下，由 $\alpha$-叠氮查尔酮、芳醛 及胺等通过多组分一锅法合成了具有较强的药理活性 的多取代咪唑类化合物苯基(1,2,4-三苯基咪唑-5-甲基酮 (Eq. 6), 该方法适用于含不同取代基的原料, 均能得到 较满意的收率(61\% 82\%).

上述反应可能的机理如下: 在三氟甲基磺酸铒的作 用下，带不同芳醛和芳胺缩合成亚胺中间体， $\alpha$-叠氮查 尔酮经过分子内成环、重排，脱去一个 $\mathrm{N}_{2}$ 分子形成负离 子中间体 $\mathbf{a}$, 氮上的孤对电子进攻亚胺化合物的亲电中 心形成中间 $\mathbf{b}$, 随后亚胺氮上的电子对进攻羰基的 $\alpha$ 位 形成二氢咪唑 $\mathbf{c}$, 最后, 在空气作用下脱去氢原子形成 目标产物取代咪唑 d (Scheme 8).
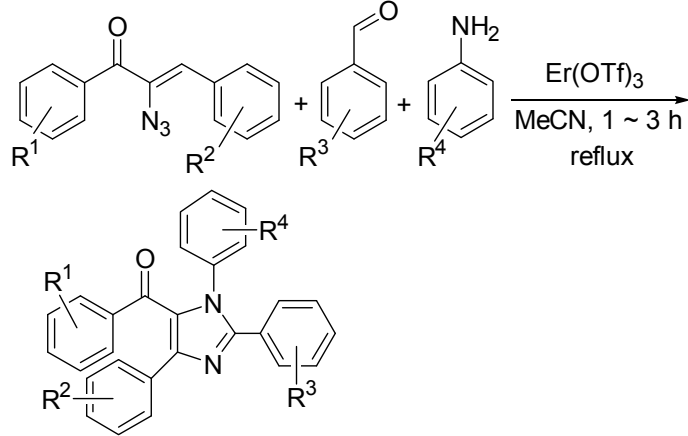

$\mathrm{R}^{1}=p-\mathrm{MeO}, p-\mathrm{NO}_{2}, p-\mathrm{Cl} ; \mathrm{R}^{2}=p-\mathrm{Cl}, m-\mathrm{Cl}, p-\mathrm{NO} ;$

$\mathrm{R}^{3}=p-\mathrm{Cl}, m-\mathrm{Cl}, p-\mathrm{NO}_{2}, p-\mathrm{CH}_{3} ; \mathrm{R}^{4}=p-\mathrm{MeO}, p-\mathrm{CF}_{3}, p-\mathrm{Cl}, p-\mathrm{Br}$

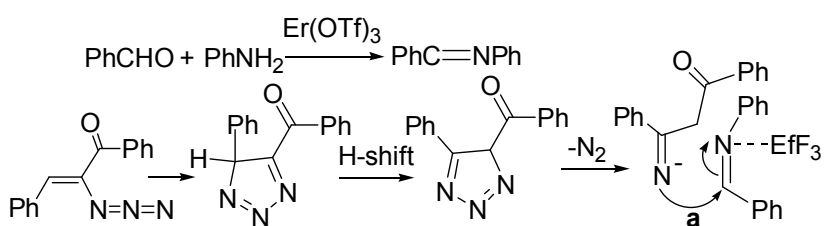<smiles>O=C(c1ccccc1)c1c(-c2ccccc2)nc(-c2ccccc2)n1-c1ccccc1</smiles><smiles>CCCC1N=C(c2ccccc2)C(C(=O)c2ccccc2)N1C(=O)C(F)(F)F</smiles><smiles>COC(=C1C(=C(F)F)C(c2ccccc2)N(C(F)(F)F)CC1c1ccccc1)c1ccccc1</smiles>

图式 8 三氟甲基磺酸铒催化合成四取代咪唑的反应机理 Scheme 8 The mechanism in formation of tetra-substituted imidazoles catalyzed by $\operatorname{Er}(\mathrm{OTf})_{3}$

2014 年, Pandya 等 ${ }^{[69]}$ 以 $\mathrm{Cu}(\mathrm{OAc})_{2} \cdot \mathrm{H}_{2} \mathrm{O}$ 为催化剂, TBHP 为氧化剂, $\mathrm{I}_{2}$ 为辅助氧化剂, 在 DMA 中, 将 $\mathrm{C}-\mathrm{H}$ 键氧化，进而形成咪唑环(Eq. 7), 产率为 $76 \%$. 该方法 反应条件温和，反应原料简单易得，是一种有效地合成 含不同官能团的 1,2,4,5-多取代咪唑的方法. 该反应可 能的机理为, 在碱的作用下, 原料发生烯醇化转变为亚 胺结构, 并发生卤代生成中间体 $\mathbf{a}$; 随后 $\mathbf{a}$ 与伯胺发生 亲核取代反应生成 $\mathbf{b}$; 在氧化剂 TBHP 作用下 $\mathbf{b}$ 脱氢形 成双亚胺结构 $\mathbf{c}$; 同时, $\mathbf{c}$ 在 Lewis 酸 $\mathrm{Cu}^{2+}$ 活化下经分子 内亲核反应成环得 $\mathbf{d}$; 最后, $\mathbf{d}$ 经过脱氢得终产物四取代 咪唑 e (Scheme 9).
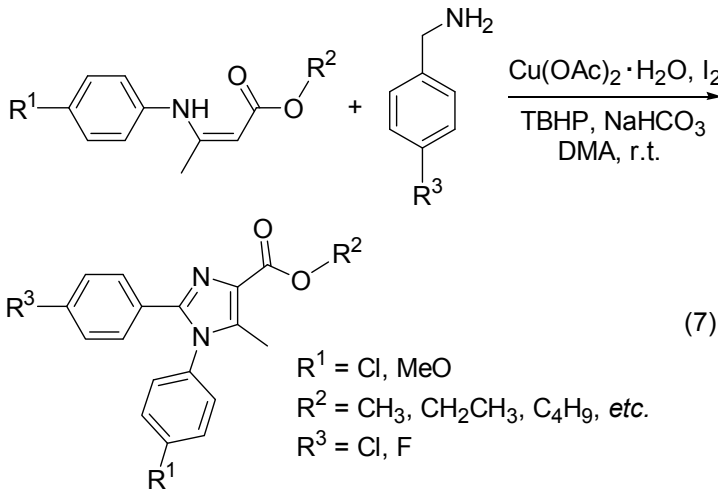


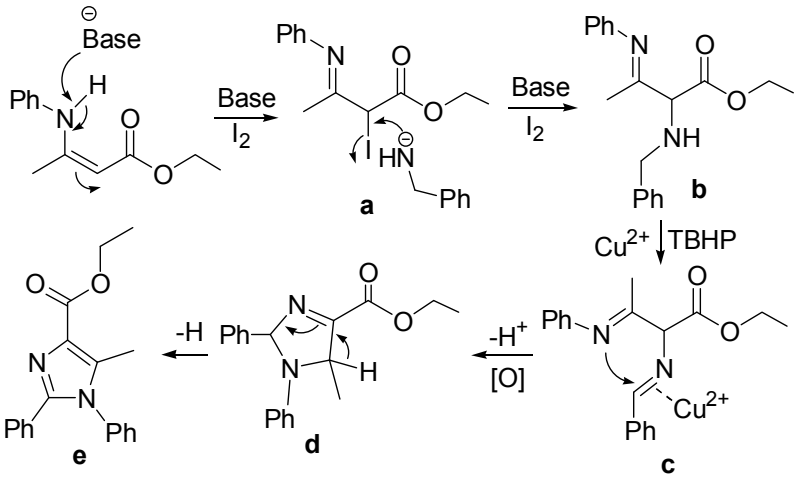

图式 9 醋酸铜催化合成四取代咪唑的反应机理

Scheme 9 The mechanism in formation of multi-substituted imidazoles catalyzed by $\mathrm{Cu}(\mathrm{OAc})_{2}$

Mamedov 等 ${ }^{[70]}$ 采用 3-芳基喹喏啉-2-酮与芳醛、乙 酸铵作用, 在乙酸-甲醇中回流, 合成了 2-芳基-4-(2-苯 并咪唑)-5-苯基咪唑(Eq. 8), 当咪唑环的 4-位存在邻苯 二胺结构时, 反应可近乎定量进行. 采用上述方法也可 以合成咪唑 $[1,5 \alpha]$ 喹喏啉-4-酮. 该方法操作简单、产品容 易分离, 为合成含多个杂环的取代咪唑提供了一种有效 的方法.

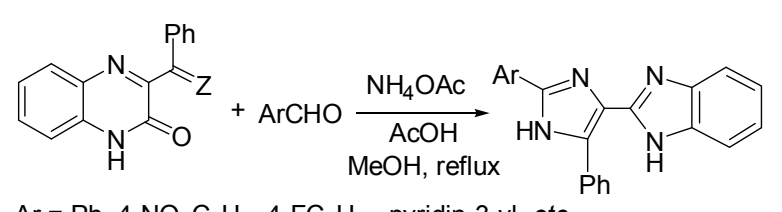

$\mathrm{Ar}=\mathrm{Ph}, 4-\mathrm{NO}_{2} \mathrm{C}_{6} \mathrm{H}_{4}, 4-\mathrm{FC}_{6} \mathrm{H}_{4}$, pyridin-3-yl, etc.

2012 年, $\mathrm{Li}$ 等 ${ }^{[71]}$ 通过呋嗡与酰氯作用形成的羰基酯 为原料, 以氧化铝负载的醋酸铵为氮源, 在无溶剂和微 波辐射条件下，合成了呋喃取代的咪唑类化合物(Eq. 9), 产率为 $49 \% \sim 90 \%$. 合成的化合物具有独特的化学发光 特性. 该方法反应时间 $(5 \sim 10 \mathrm{~min})$ 短, 操作方便, 为合 成含氮杂环化合物提供了高效便捷的途径.

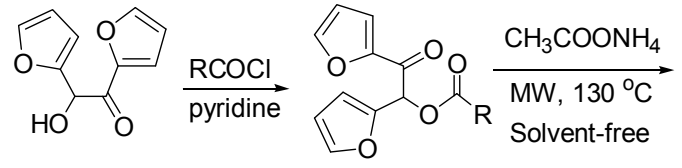

R: Alkyl, Aryl, Substituted aryl<smiles></smiles>

Mloston 等 ${ }^{[72]}$ 利用外消旋的 $\beta$-氨基- $\alpha$-三氟甲基醇、 邻羰基肟及甲醛作用，合成了外消旋的含三氟甲基的三 取代咪唑(Eq. 10). 选用光学纯的原料(如 $S$ 型)进行上述 反应，可以顺利生成旋光性保持的三取代咪唑产物(如 $S$ 型), 提供了一种合成含有光学活性的基团的三取代咪
唑类化合物的有效方法.

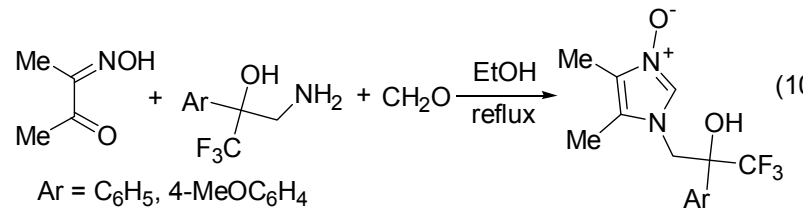

\section{3 由畨或氨基腈和取代芳烯烃为底物合成多取 代咪唑}

Liu 等 ${ }^{[73]}$ 利用硝基烯烃化合物与 $N$-芳基苯甲脒等 为原料, 在铁(III)催化下, 在 $\mathrm{DMF}$ 中加热到 $90{ }^{\circ} \mathrm{C}$, 经过 $3+2$ 环加成反应, 合成了 $1,3,5$-三取代咪唑类化 合物(Eq. 11), 产率最高可达 86\%. 该方法操作方便, 环境友好，区域选择性高，是一种合成多取代咪唑类 化合物的有效方法. 该反应可能的机理为: $N$-芳基苯 甲榺在 Lewis 酸 $\mathrm{FeCl}_{3}$ 活化下和硝基烯烃化合物发生 Michael 加成形成中间体 $\mathbf{a}, \mathbf{a}$ 在 $\mathrm{FeCl}_{3}$ 作用下发生分子 内亲核加成生成中间体 $\mathbf{b}$, 进而失水一分子次硝酸和 一分子水得到产物三取代咪唑 $\mathbf{c}$ (Scheme 10).
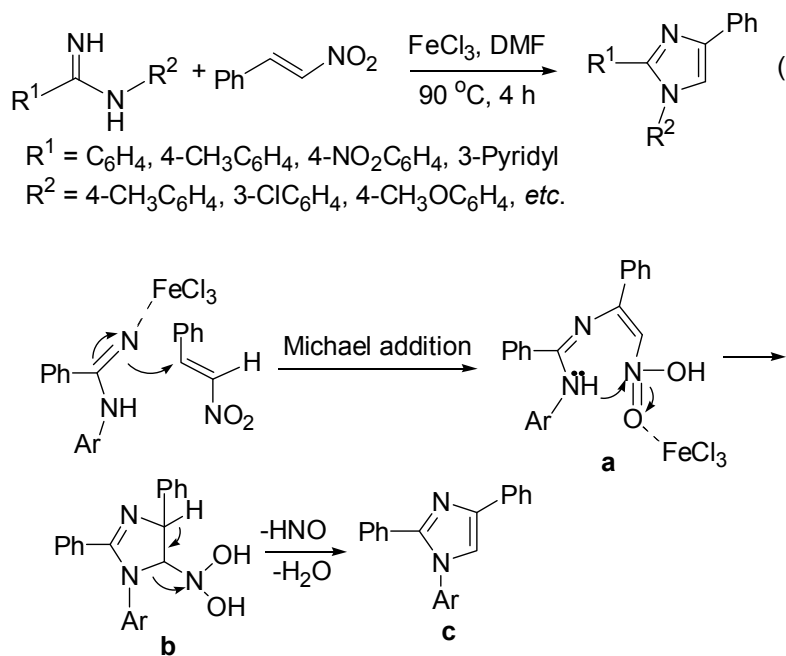

图式 $10 \mathrm{FeCl}_{3}$ 催化合成 1,2,4-三取代咪唑的反应机理 Scheme 10 The mechanism in formation of 1,2,4-trisubstituted imidazoles catalyzed by $\mathrm{FeCl}_{3}$

Tang 等 ${ }^{[74]}$ 通过 $N$-苯基-苯甲脒与硝基芳乙烯为原 料, 以碘化亚铜一联吡啶为催化体系, 在氧气存在下, 通 过 3+2 环加成反应合成了 1,2,4,5-四取代咪唑, 产率为 58\% 75\% (Eq. 12). 碘化亚铜-联吡啶体系催化效率较 高且选择性好，该反应利用氧气作为氧化剂既经济又环 保，丰富了从简单易得的原料反应制备多取代咪唑的方 法.

类似地, Guo 等 ${ }^{[75]]}$ 以取代的胺与 2-氰基-3-甲基吡啶 作用生成的苯甲脒为起始原料，在异丙醇中与 3-溴丙酸 乙酯反应合成了 1,2,4-三取代咪唑(Scheme 11), 产率最 
高可达 $85 \%$. 所得化合物具有较强的抑制细胞苂光素酶 的活性.
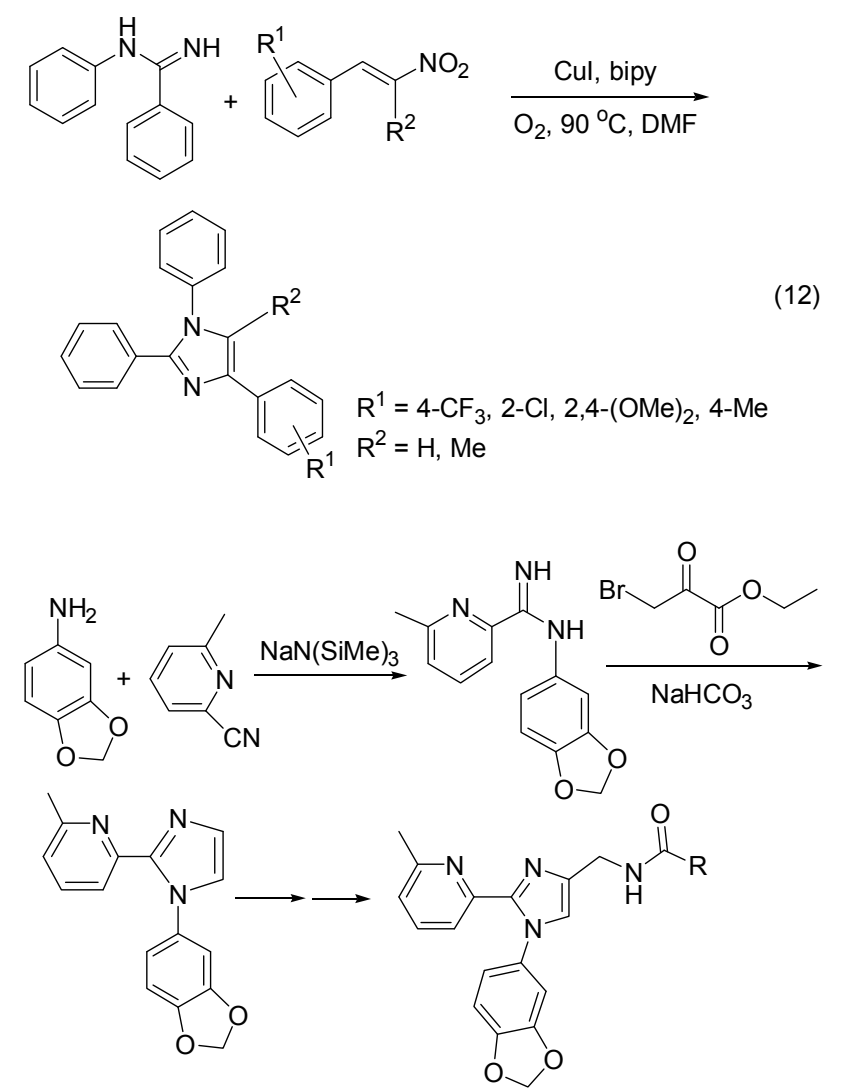

$\mathrm{R}$ = amino, isopropylamino, morpholino

图式 11 以胺和睛为原料合成 1,2,4-三取代咪唑

Scheme 11 Synthesis of 1,2,4-trisubstituted imidazoles from amine and nitrile

2014 年, Pusch ${ }^{[76]}$ 发展了一种利用 $\alpha$-氨基腈、醛和异 噁唑在紫外光促进下合成 1-酰甲基-四取代咪唑化合物 的方法，产率为 24\% 83\% (Eq. 13). 通过密度泛函理论 (DFT)法计算反应所经过的过渡态的活化能和等位面等, 发现反应过程中的关键步骤涉及碳负离子的亲核加成 和氮杂环丙烯中 $\mathrm{C}^{2}-\mathrm{C}^{3}$ 化学键的断裂. 以二苯基异噁 唑、氨基乙腈以及 4-氧基苯甲醛之间的反应为例, 根据 已有的实验事实和 DFT 法计算的结果，可以推断该反 应可能的反应机理如下: 异噁唑在紫外光的作用下开环 生成酰基氮杂环丙烯, 芳醛与氨基腈缩合生成亚胺负离 子; 氮杂环丙烯与亚胺碳负离子发生亲核加成生成带负 电荷的中间体 $\mathbf{a} ; \mathbf{a}$ 发生分子内亲核加成反应关环生成氮 负离子中间体 $\mathbf{b}, \mathbf{b}$ 脱去氧基负离子生成中间体 $\mathbf{c}$; 在碱 存在下, $\mathbf{c}$ 脱去一分子水形成碳负离子中间体 $\mathbf{d}$; 随后, $\mathbf{d}$ 内三元环的 $C^{2}-C^{3}$ 键断开形成含烯醇负离子的咪唑环 $\mathbf{e}$; 最后 $\mathrm{e}$ 中烯醇式结构经过互变异构转化为酮式产物 (Scheme 12).

$$
\text { 每 } \begin{aligned}
& \mathrm{KOBu}-t, \mathrm{hv} \\
& \mathrm{MeCN} \text {, r.t., } 6 \mathrm{~h}
\end{aligned}
$$

$\mathrm{R}^{1}=\mathrm{Ph}, p-\mathrm{F}-\mathrm{C}_{6} \mathrm{H}_{4} ; \mathrm{R}^{2}=\mathrm{Ph}, p-i-\mathrm{Pr}-\mathrm{C}_{6} \mathrm{H}_{4} ; \mathrm{R}^{3}=\mathrm{Ph}, p-\mathrm{F}-\mathrm{C}_{6} \mathrm{H}_{4}, \mathrm{Me}, \mathrm{Cy}$ $\mathrm{R}_{4}=p-\mathrm{F}-\mathrm{C}_{6} \mathrm{H}_{4}, p-\mathrm{NO}_{2}-\mathrm{C}_{6} \mathrm{H}_{4}, p-\mathrm{NC}-\mathrm{C}_{6} \mathrm{H}_{4}, p-\mathrm{F}_{3} \mathrm{CC}_{6} \mathrm{H}_{4}, 4-p y r i d y l$

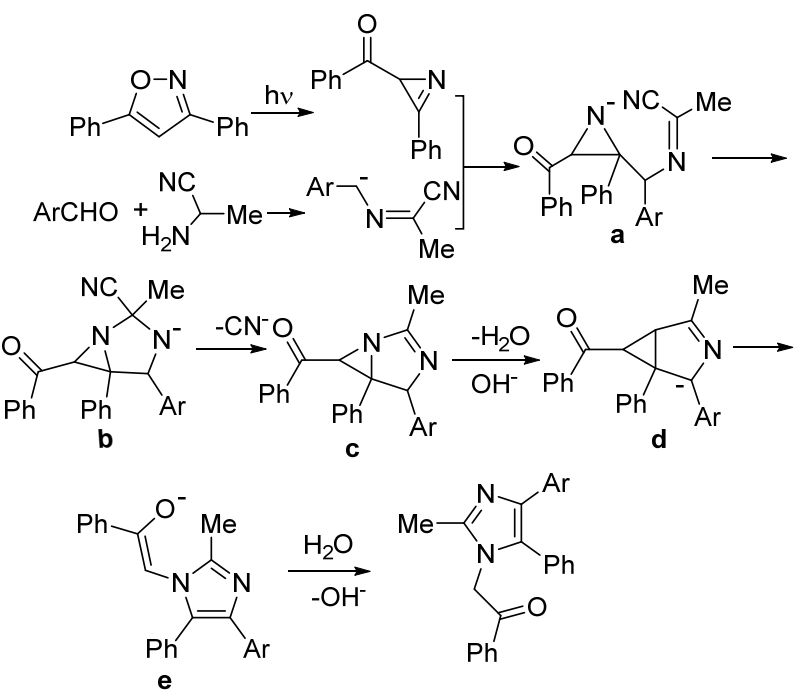

图式 12 紫外光促进从异噁唑合成四取代咪唑的反应机理 Scheme 12 The mechanism in formation of tetra-substituted imidazoles from isooxazole promoted by ultraviolet rays

\section{4 由 2-卤代酮为底物合成多取代咪唑}

Mehdi 等 ${ }^{[77]}$ 以 $\alpha$-澳代芳乙酮、伯胺、芳酫和醋酸铵 为原料，在无溶剂条件下，于 $130{ }^{\circ} \mathrm{C}$ 反应 $2 \mathrm{~h}$ ，合成了一 系列 1,2,4-三取代咪唑(Eq. 14), 产率为 84\% 95\%. 该 方法原料简单易得，无需溶剂和催化剂，反应时间短， 产率高，是一种理想的合成功能化咪唑的方法. 该反应 的机理为如下: 伯胺与醛缩合生成亚胺, 亚胺与 2-溴代 芳乙酮作用生成亚胺正离子 $\mathbf{a} ; \mathbf{a}$ 与氨发生加成反应生成 缩醛胺 $\mathbf{b}$; 随后, $\mathbf{b}$ 的氨基部分与邻近的羰基发生缩合反 应，脱去一分子水形成二氢咪唑中间体 $\mathbf{c}$ ，进一步在空 气存在下，脱氢得产物 1,2,4-三取代咪唑 $\mathbf{d}$ (Scheme 13).

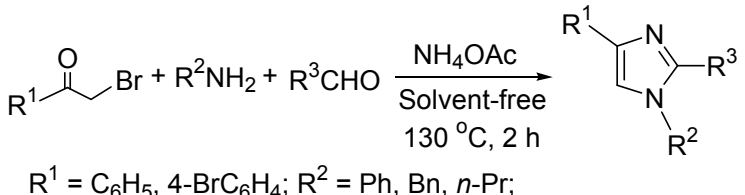

$\mathrm{R}^{1}=\mathrm{C}_{6} \mathrm{H}_{5}, 4-\mathrm{BrC}_{6} \mathrm{H}_{4} ; \mathrm{R}^{2}=\mathrm{Ph}, \mathrm{Bn}, n-\mathrm{Pr}$

$\mathrm{R}^{3}=\mathrm{C}_{6} \mathrm{H}_{5}, 4-\mathrm{ClC}_{6} \mathrm{H}_{4}, 4-\mathrm{CH}_{3} \mathrm{C}_{6} \mathrm{H}_{4}, 4-\mathrm{NO}_{2} \mathrm{C}_{6} \mathrm{H}_{4}$, etc

\section{5 由酰氯和亚胺为底物合成多取代咪唑}

2006 年, Siamaki 等 ${ }^{[78]}$ 在自制的钯催化剂作用下, 酰 氯与两分子不同取代的亚胺(一个亚胺为对甲苯磺酰基 


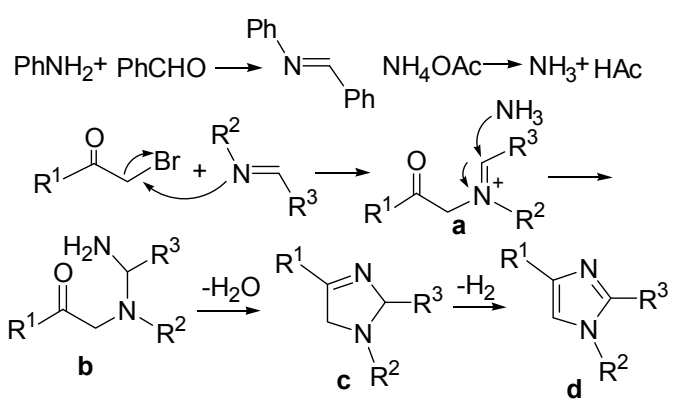

图式 13 以溴代芳乙酮为底物合成 $1,2,4$-三取代咪唑的反应 机理

Scheme 13 The mechanism in formation of 1,2,4-trisubstituted imidazoles from bromoarylethanone

取代, 另一个为烃基取代)作用, 在一定压力的一氧化碳 存在下，合成了多取代咪唑，产率为 $60 \% \sim 76 \%$. 该反 应具有很高的选择性: 在反应中烃基取代的亚胺与酰氯 反应，而磺酰基取代的胺不会与酰氯作用，原因是磺酰 基对反应具有钝化作用. 该方法所用原料所含基团的选 择性广, 为合成用其他方法难以合成的功能分子提供了 便捷的合成方法. 例如，一种 p 38 MAP 激活酶拟制剂的 合成(Eq. 15), 其 2,4,5 位三个取代基均不相同, 用以前 的方法其原料不易获得，但采用本方法，则非常简单方 便.

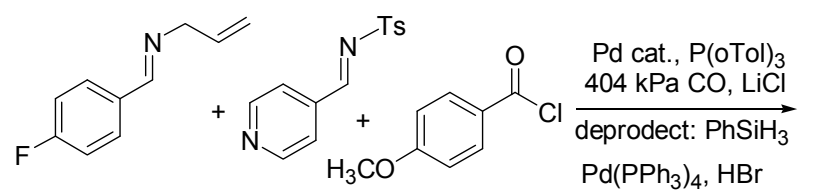<smiles>Oc1ccc(-c2nc(-c3ccncc3)c(-c3ccc(F)cc3)[nH]2)cc1</smiles>

该反应过程的机理如下：亚胺与酰氯发生亲核取代 反应生成亚胺盐中间体 $\mathbf{a}$; 中间体 $\mathbf{a}$ 会在加热或对甲苯 苯磺酰基存在下生成副产物 $\mathbf{g}$ 和 $\mathbf{h}$, 导致产率降低. 考 虑到亚胺盐中间体分解是热力学控制过程, 因而通过降 低反应温度, 增加 $\mathrm{CO}$ 的压力, 达到抑制副反应的目的. 另外, 在反应体系中加入膦配体 $\mathrm{P}(o-\mathrm{Tol})_{3}$, 会增加催化 剂的催化效率; 同时, 加入无机盐如 $\mathrm{LiCl}$ 也能影响亚胺 盐中间体分解的平衡，使其向左移动. 亚胺盐中间体 $\mathbf{a}$ 与钯催化剂配位形成配合物中间体 $\mathbf{b}$; 一氧化碳取代配 合物中的膦配体与钯配位生成中间体 $\mathbf{c} ; \mathbf{c}$ 经过一系列变 化脱去一分子 $\mathrm{HCl}$, 同时脱去 $\mathrm{Pd}($ 重新进入循环)形成噁 唑酮中间体 $\mathbf{f} ; \mathbf{f}$ 与 $\mathrm{Ts}$ 取代的亚胺作用, 脱去一分子 $\mathrm{CO}_{2}$ 和 Ts 形成产物多取代咪唑(Scheme 14).

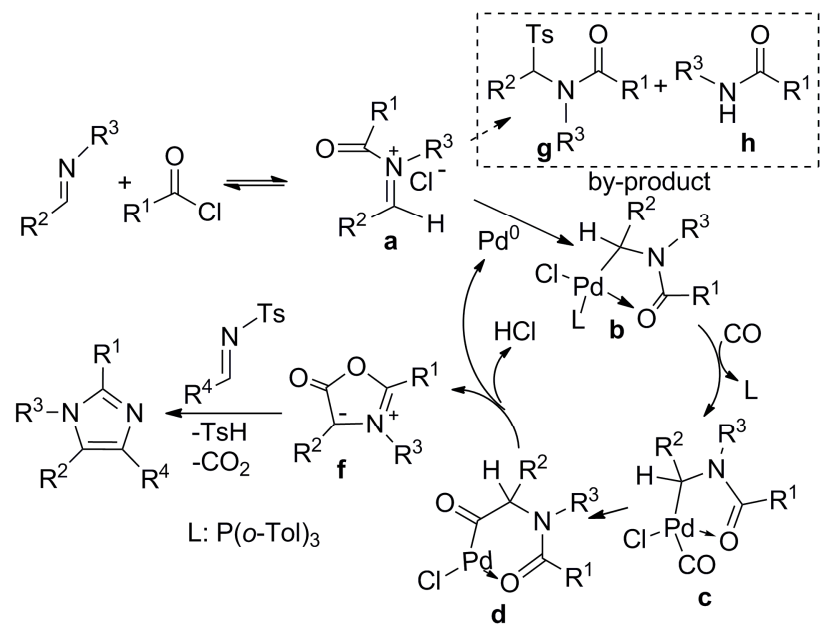

图式 14 从亚胺及酰氯合成四取代咪唑的反应机理

Scheme 14 The mechanism in formation of tetra-substituted imidazoles from imines and acid chlorides

\section{6 以炔类化合物为底物合成多取代咪唑}

2012 年, Jiang 等 ${ }^{[79]}$ 利用炔丙基胺、硫酰叠氮化合物 和端基炔为原料, 在 $\mathrm{CuI} / \mathrm{Et}_{3} \mathrm{~N}$ 和 $\mathrm{Cs}_{2} \mathrm{CO}_{3} / \mathrm{MeCN}$ 体系催 化下，通过 “两步一锅法”，经过烯酮亚胺(ketenimine) 和氨基丙二烯中间体，合成了四取代咪唑，产率达 $39 \% \sim 83 \%$ (Eq. 16). 整个反应过程条件温和，操作简 便. 反应分为两步进行：首先，在低价铜诱导下，硫酰 叠氮化合物和端基炔经过环加成 $(\mathrm{CuACC}$, copper catalyzed azide-alkyne cycloaddition)过程, 就地生成 $N$-磺酰 烯酮亚胺中间体 $\mathbf{a}$; 进而, 炔丙基胺中的氮原子作为亲 核基团进攻烯酮亚胺 $\mathbf{a}$ 的中心碳生成中间体 $\mathbf{b}$; 随后， 在碳酸铯(作为碱)的作用下, $\mathbf{b}$ 中的炔丙基部分转化为丙 二烯结构 $\mathbf{c}, \mathbf{c}$ 经环化形成具有共振结构的 $\mathbf{d}$ 和 $\mathbf{e}$; 最后, $\mathbf{e}$ 经过磺酰基的 1,3-迁移形成产物四取代咪唑(Scheme 15).

$$
\text { }
$$

2013 年, Chen 等 ${ }^{[80]}$ 在有机催化剂特戊酸(三甲基乙 酸)催化下, 通过二取代乙炔、芳醛、胺和乙酸铵等一锅 法合成了三取代和四取代咪唑，产率可达 $72 \%$ ～87\% (Scheme 16). 在反应过程中, 在特戊酸和 DMSO 的存在 下，炔被氧化成二羰基化合物，后面的反应过程与邻二 酮为底物合成多取代咪唑，存在完全相同的反应机理, 


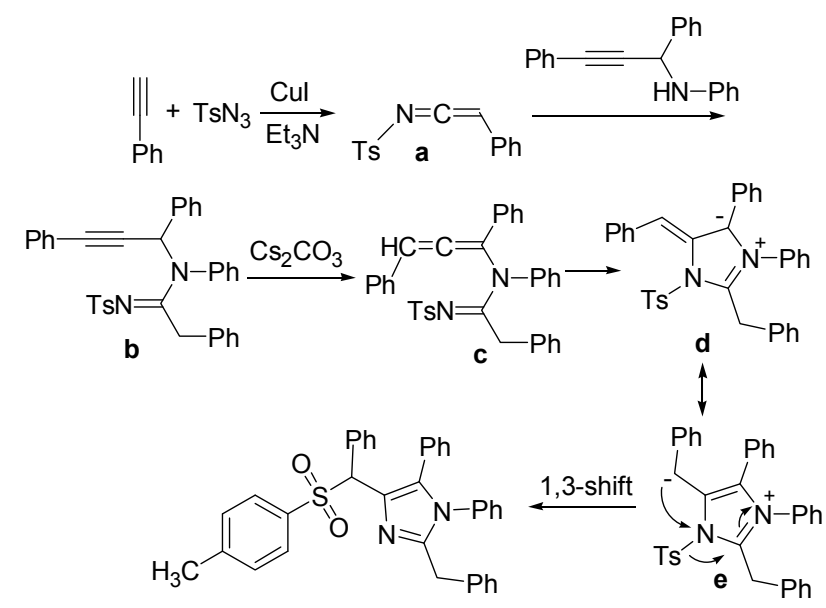

图式 15 从炔胺、炔及磺酰基叠氮化合物合成三取代咪唑的 反应机理

Scheme 15 The mechanism in formation of tri-substituted imidazoles from propargyl amines, sulfonyl azides and alkynes

$$
\begin{aligned}
& \mathrm{R}^{1}=\mathrm{R}^{2}+\mathrm{R}^{3} \mathrm{CHO} \frac{\substack{\text { pivOH, } 140{ }^{\circ} \mathrm{C} \\
\text { DMSO: } \mathrm{H}_{2} \mathrm{O}}}{\mathrm{NH}_{4} \mathrm{OAc}} \mathrm{R}^{2} \\
& \mathrm{R}^{1}=\mathrm{R}^{2}+\mathrm{R}^{3} \mathrm{CHO}+\mathrm{R}^{4} \mathrm{NH}_{2} \frac{\substack{\text { pivOH, } 140{ }^{\circ} \mathrm{C} \\
\mathrm{DMSO}: \mathrm{H}_{2} \mathrm{O}}}{\mathrm{NH}_{4} \mathrm{OAc}} \mathrm{R}^{2} \\
& \mathrm{R}^{1} \text { or } \mathrm{R}^{2}=\mathrm{Ph}, 4-\mathrm{MeOC}_{6} \mathrm{H}_{4}, 3,4,5-(\mathrm{MeO})_{3} \mathrm{C}_{6} \mathrm{H}_{2} \\
& \mathrm{R}^{3}=\mathrm{Ph}, \mathrm{CH}_{3} \mathrm{OC}_{6} \mathrm{H}_{4} \text {, 2-furyl, } \mathrm{H}, \mathrm{C}_{2} \mathrm{H}_{5},\left(\mathrm{CH}_{3}\right)_{2} \mathrm{CH} \\
& \mathrm{R}^{4}=\mathrm{Ph}, 4-\mathrm{BrC}_{6} \mathrm{H}_{4}, \mathrm{CH}_{3}
\end{aligned}
$$

图式 16 非金属催化剂促进从取代炔合成多取代咪唑

Scheme 16 Synthesis of multi-substituted imidazoles from internal alkynes promoted by metal-free catalyst

具体过程见 Schemes 2 3. 该方法拓展了以邻二酮为反 应底物合成多取代咪唑的范围，可以合成不同结构的多 取代咪唑类化合物，采用芳醛作原料收率 $(83 \%$ ～87\%) 比脂肪醛 $(68 \% \sim 74 \%)$ 略高.

\section{7 总结和展望}

本文归纳了以 1,2 -二酮或 $\alpha$-差基酮、二酮衍生物、 脒或氨基腈、卤代酮、酰氯和亚胺, 以及炔类化合物等 为反应底物合成多取代咪唑的几种方法, 其中通过 1,2二酮或 $\alpha$-羟基酮为底物合成标题化合物的方法报道最 多. 从反应手段上看，除了传统的加热以外，微波辐射、 超声波辐射以及太阳光照射等手段在合成多取代咪唑 中也得到一定程度的应用, 使多取代咪唑的合成变得更 加简洁、绿色、高效. 同时, 无溶剂反应法或离子液体 作为反应介质应用于多取代咪唑的合成中，克服了传统 的有机溶剂易挥发、易污染环境、造价高等缺点, 使多 取代咪唑的合成更符合现代有机合成的理念.

合成多取代咪唑所用的催化剂从质子酸、Lewis 酸、
离子液体等均相催化剂, 发展到负载型异相催化剂. 负 载型催化剂具有绿色环保、可以循环使用等优势，越来 越普遍地应用于多取代咪唑的合成中. 传统的负载材料 是无机、高分子材料，由于支撑材料的位阻和扩散作用， 反应物难以接近催化中心，导致活性成分负载后催化活 性降低. 但是纳米催化剂的出现, 改变了这种局面, 这 是由于纳米材料存在特殊的表面效应和小尺寸效应，因 而大大提高了催化效率. 纳米催化剂的使用, 成为今后 有机合成重要的发展趋势之一．此外，近年来，不含金 属的催化剂尤其是有机催化剂，因其低毒、高效等优点, 在多取代咪唑的合成中占有越来越重要的地位.

综上所述，多组分反应在包括多取代咪唑在内的杂 环化合物的合成中占有越来越重要的地位. 相信随着广 大化学工作者的努力，更多的绿色、高效新颖的反应方 法、催化剂以及反应手段会越来越多地应用于多取代咪 唑类化合物的合成中.

\section{References}

[1] Koch, P.; Bauerlein, C.; Jank, H.; Laufer, S. J. Med. Chem. 2008, 51,5630 .

[2] Li, W. J.; Li, Q.; Liu, D. L.; Ding, M. W. J. Agric. Food Chem. 2013, 61, 1419.

[3] Xu, Z. X.; Wang, S. M.; Zhao, L.; Zhang, S. L.; Li, J. B. Chin. J. Org. Chem. 2003, 23(9), 950 (in Chinese).

(许祖勋, 王世敏, 赵雷, 张胜利, 黎俊波, 有机化学, 2003 23(9), 950.)

[4] Yang, W. H.; Xiao, G. M.; Kong, X. X. Appl. Chem. 2003, 20(4), 406 (in Chinese). (杨为华，肖国民，孔祥翔，应用化学, 2003, 20(4), 406.)

[5] Dupont, J.; de Souza, R. F.; Suarez, P. A. Z. Chem. Rev. 2002, 102, 3667.

[6] Ye, H.; Wang, W.; Zhu, X.; Chen, W.; Xie, L.; Yuan, Y. Chin. J. Org. Chem. 2013, 33, 827 (in Chinese).

(叶宏民, 王薇, 朱晓晓, 陈伟强, 谢莉莉, 袁耀锋, 有机化学, 2013, 33, 827.)

[7] Bian, L.; Zeng, X.; He, R.; Luo, C.; Lin, Z. Chin. J. Org. Chem. 2014, 34, 994 (in Chinese)

(市垒，曾向潮，何如，罗创龙，林志强，有机化学，2014，34, 994.)

[8] Gao, X.; Fan, J.; Wang, X.; Zhang, Y. Acta Chim. Sinica 2013, 71, 1411 (in Chinese) (高霞, 樊静, 王小龙 张艳树, 化学学报, 2013, 71, 1411.)

[9] Bourissou, D.; Guerret, O.; Gabbai, F. P.; Bertrand, G. Chem. Rev. 2000, 100, 39

[10] Climent, M. J.; Corma, A.; Iborra, S. RSC Adv. 2012, 2, 16.

[11] Domling, A. Chem. Rev. 2006, 106, 17

[12] Cao, S.; Jing, Y.; Liu, Y.; Wan, J. Chin. J. Org. Chem. 2014, 34, 876 (in Chinese)

(曹硕, 景艳锋, 刘云云, 万结平, 有机化学, 2014, 34, 876.)

[13] Tang, M.; Xing, D.; Cai, M.; Hu, W. Chin. J. Org. Chem. 2014, 34, 1268 (in Chinese)

(唐敏, 邢栋, 蔡茂强, 胡文浩, 有机化学, 2014, 34, 1268.)

[14] Radziszewski, B. Chem. Ber. 1882, 15, 1493.

[15] Japp, F.; Robinson, H. Chem. Ber. 1882, 15, 1268.

[16] Karimi-Jaberi, Z.; Barekat, M. Chin. Chem. Lett. 2010, 21(10), 
1183.

[17] Joshi, R. S.; Mandhane, P. G.; Shaikh, M. U.; Kale, R. P.; Gill, C. H. Chin. Chem. Lett. 2010, 21(4), 429.

[18] Pasha, M. A.; Nizam, A. J. Saudi Chem. Soc. 2011, 15(1), 55

[19] Das, B.; Kashanna, J.; Kumar, R. A.; Jangili, P. Monatsh. Chem. 2013, 144 (2), 223.

[20] Sharma, S. D.; Hazarika, P.; Konwar, D. Tetrahedron Lett. 2008, 49(14), 2216.

[21] Reddy, M. V.; Jeong, Y. T. J. Fluorine Chem. 2012, 142, 45.

[22] Khosropour, A. R. Ultrason. Sonochem. 2008, 15(5), 659.

[23] Sangshetti, J. N.; Kokare, N. D.; Kotharkar, S. A.; Shinde, D. B. Chin. Chem. Lett. 2008, 19,762.

[24] Wang, L.-M.; Wang, Y.-H.; Tian, H.; Yao, Y.-F.; Shao, J.-H.; Liu, B. J. Fluorine Chem. 2006, 127(12), 1570.

[25] Shen, M.-G.; Cai, C.; Yi, W.-B. J. Fluorine Chem. 2008, 129(6), 541

[26] Karami, B.; Dehghani, F. M.; Eskandari, K. Croat. Chem. Acta 2012, 85(2), 147.

[27] Rajanarendar, E.; Murthy, K. R.; Reddy, M. N. Indian J. Chem. 2011, $50 B, 926$

[28] Kidwai, M.; Mothsra, P.; Bansal, V.; Somvanshi, R. K.; Ethayathulla, A. S.; Dey, S.; Singh, T. P. J. Mol. Catal. A: Chem. 2007, 265(1 2), 177.

[29] Wang, X. C.; Gong, H. P.; Quan, Z. J.; Li, L.; Ye, H. L. Chin. Chem. Lett. 2009, 20(1), 44

[30] Chary, M. V.; Keerthysri, N. C.; Vupallapati, S. V. N.; Lingaiah, N.; Kantevari, S. Catal. Commun. 2008, 9(10), 2013.

[31] Murthy, S. N.; Madhav, B.; Nageswar, Y. V. D. Tetrahedron Lett. 2010, $51(40), 5252$.

[32] Ramesh, K.; Murthy, S. N.; Karnakar, K.; Nageswar, Y. V. D.; Vijayalakhshmi, K.; Devi, B. L. A. P.; Prasad, R. B. N. Tetrahedron Lett. 2012, 53(9), 1126.

[33] Samai, S.; Nandi, G. C.; Singh, P.; Singh, M.S. Tetrahedron 2009, 65(49), 10155.

[34] Nagargoje, D.; Mandhane, P.; Shingote, S.; Badadhe, P.; Gill, C. Ultrason. Sonochem. 2012 19, 94.

[35] Mahajan, A.; Aulakh, R. K.; Bedi , R. K.; Kumar, S.; Kumar, S.; Aswal, D. K. Synth. Met. 2012, 162, 58.

[36] Martorana, A.; Pace, A.; Buscemi, S.; Piccionello, A. P. Org. Lett. 2012, 14(13), 3240.

[37] Xue, W.-J.; Li, H.-Z.; Gao, F.-F.; Wu, A. Tetrahedron 2014, 70(2), 239.

[38] Zang, H.; Su, Q.; Mo, Y.; Cheng, B.-W.; Jun, S. Ultrason. Sonochem. 2010, 17(5), 749 .

[39] Siddiqui, S. A.; Narkhede, U. C.; Palimkar, S. S.; Daniel, T.; Lahoti, R. J.; Srinivasan K. V. Tetrahedron 2005, 61(14), 3539.

[40] Xia, M.; Lu, Y.-D. J. Mol. Catal. A: Chem. 2007, 265(1 2), 205.

[41] Shaterian, H. R.; Ranjbar, M. J. Mol. Liq. 2011, 160 (1), 40.

[42] Zolfigol, M. A.; Khazaei, A.; Moosavi-Zare, A. R.; Zare, A.; Asgari, Z.; Khakyzadeh, V.; Hasaninejad, A. J. Ind. Eng. Chem. 2013, 19(3), 721.

[43] MaGee, D. I.; Bahramnejad, M.; Dabiri, M. Tetrahedron Lett. 2013, 54(21), 2591.

[44] Jourshari, M. S.; Mamaghani, M.; Shirini, F.; Tabatabaeian, K.; Rassa, M.; Langari, H. Chin. Chem. Lett. 2013, 24(11), 993.

[45] Heravi, M. M.; Derikvand, F.; Bamoharram, F. F. J Mol. Catal. A: Chem. 2007, 263(1 2), 112.

[46] Nagarapu, L.; Apuri, S.; Kantevari, S. J Mol. Catal. A: Chem. 2007, 266(1 2), 104

[47] Fantini, M.; Zuliani, V.; Spotti, M. A.; Rivara, M. J. Comb. Chem. 2010, $12(1), 181$.
[48] Heravi, M. M.; Bakhtiari, K.; Oskooie, H. A.; Taheri, S. J. Mol. Catal. A: Chem. 2007, 263(1 2), 279.

[49] Kannan, V.; Sreekumar, K. J. Mol. Catal. A: Chem. 2013, 376, 34.

[50] Kantevari, S.; Vuppalapati, S. V. N.; Biradar, D. O.; Nagarapu. L. J. Mol. Catal. A: Chem. 2007, 266(1 2), 109.

[51] Karimi, A. R.; Alimohammadi, Z.; Azizian, J.; Mohammadi, A. A.; Mohammadizadeh, M. R. Catal. Commun. 2006, 7(9), 728.

[52] Niknam, K.; Deris, A.; Naeimi, F.; Majleci, F. Tetrahedron Lett. 2011, 52(36), 4642.

[53] Mukhopadhyay, C.; Tapaswi, P. K.; Drew, M. G. B. Tetrahedron Lett. 2010, 51(30), 3944.

[54] Sivakumar, K.; Kathirvel, A.; Lalitha, A. Tetrahedron Lett. 2010, $51(22), 3018$.

[55] Gupta, P.; Paul, S. J. Mol. Catal. A: Chem. 2012, 352, 75

[56] Mohammadi, A.; Keshvari, H.; Sandaroos, R.; Maleki, B.; Rouhi, H.; Moradi, H.; Sepehr, Z.; Damavandi, S. Appl. Catal., A: Gen. 2012, 429 430, 73 .

[57] Mekheimer, R. A.; Hameed, A. M. A.; Mansour, S. A. A.; Sadek, K. U. Chin. Chem. Lett. 2009, 20(7), 812.

[58] Xiao, L. W.; Gao, H. J.; Kong, J.; Liu, G. X.; Li, L. L.; Duan, J. D. Chin. J. Org. Chem. 2014, 34(12), 2511 (in Chinese).

(肖立伟, 高红杰, 孔洁, 刘光仙, 李玲玲, 段敬丹, 有机化学, 2014, 34(12), 2511.)

[59] Safari, J.; Zarnegar, Z. C. R. Chim. 2013, 16(10), 920.

[60] Safari, J.; Zarnegar, Z. Ultrason. Sonochem. 2013, 20(2), 740.

[61] Safari, J.; Gandomi-Ravandi, S.; Akbari, Z. J. Adv. Res., 2013, 4(6), 509.

[62] Teimouri, A.; Chermahini, A. N. J. Mol. Catal. A: Chem. 2011, $346(1 \sim 2), 39$.

[63] Ray, S.; Das, P.; Bhaumik, A.; Dutta, A.; Mukhopadhyay, C. Appl. Catal., A: Gen. 2013, 458, 183.

[64] Keivanloo, A.; Bakherad, M.; Imanifar, E.; Mirzaee, M. Appl. Catal., A: Gen. 2013, 467, 291.

[65] Mirjalili, B. F.; Bamoniri, A. H.; Zamani, L. Sci. Iran. 2012, 19(3), 565 .

[66] Aziizi, N.; Manochehri, Z.; Nahayi, A.; Torkashvand, S. J. Mol. Liq. 2014, 196, 153.

[67] Rostamnia, S.; Zabardasti, A. J. Fluorine Chem. 2012, 144, 69.

[68] Rajaguru, K.; Suresh, R.; Mariappan, A.; Muthusubramanian, S.; Bhuvanesh, N. Org. Lett. 2014, 16(3), 744.

[69] Pandya, A. N.; Agrawal, D. K. Tetrahedron Lett. 2014, 55(10), 1835.

[70] Mamedov, V. A.; Zhukova, N. A.; Beschastnova, T. N.; Gubaidullin, A. T.; Rakov, D. V.; Rizvanov, I. K. Tetrahedron Lett. 2011, 52(33), 4280.

[71] Li, B.; Gu, Q.; He, Y.; Zhao, T.; Wang, S.; Kang, J.; Zhang, Y. C. R. Chim. 2012, 15(9), 784.

[72] Mlostoń, G.; Obijalska, E.; Heimgartner, H. J. Fluorine Chem. 2011, 132(11), 951.

[73] Liu, X.; Wang, D.; Chen, B. Tetrahedron 2013, 69(45), 9417.

[74] Tang, D.; Wu, P.; Liu, X.; Chen, Y.-X.; Guo, S.-B.; Chen, W.-L.; Li, J.-G.; Chen, B.-H. J. Org. Chem. 2013, 78(6), 2746.

[75] Guo, C.; Zhang, C.; Li, X.; Li, W.; Xu, Z.; Bao, L.; Ding, Y.; Wang, L.; Li, S. Bioorg. Med. Chem. Lett. 2013, 23(21), 5850.

[76] Pusch, S.; Opatz, T. Org. Lett. 2014, 16, 5430.

[77] Mehdi, A.; Samira, A.; Shahzad, F.; Jafar, A. D.; Peiman, M. Synlett 2009, 3263.

[78] Siamaki, A. R.; Arndtsen, B. A. J. Am. Chem. Soc. 2006, 128, 6050.

[79] Jiang, Z; Lu, P.; Wang, Y. Org. Lett. 2012, 14(24), 6266.

[80] Chen, C.-Y.; Hu, W.-P.; Yan, P.-C.; Senadi, G. C.; Wang, J.-J. Org. Lett. 2013, 15(24), 6116.

(Cheng, F.) 